\title{
Edge states and conformal boundary conditions in super spin chains and super sigma models
}

\author{
Roberto Bondesan ${ }^{1,2}$, Jesper L. Jacobsen ${ }^{1,3}$ and Hubert Saleur ${ }^{2,4}$ \\ ${ }^{1}$ LPTENS, École Normale Supérieure, 24 rue Lhomond, 75231 Paris, France \\ ${ }^{2}$ Institute de Physique Théorique, CEA Saclay, \\ F-91191 Gif-sur-Yvette, France \\ ${ }^{3}$ Université Pierre et Marie Curie, 4 place Jussieu, 75252 Paris, France \\ ${ }^{4}$ Physics Department, USC, Los Angeles, CA 90089-0484, USA
}

\begin{abstract}
The sigma models on projective superspaces $\mathbb{C P}^{N+M-1 \mid N}$ with topological angle $\theta=\pi \bmod 2 \pi$ flow to non-unitary, logarithmic conformal field theories in the low-energy limit. In this paper, we determine the exact spectrum of these theories for all open boundary conditions preserving the full global symmetry of the model, generalizing recent work on the particular case $M=0$ [C. Candu et al, JHEP02(2010)015]. In the sigma model setting, these boundary conditions are associated with complex line bundles, and are labelled by an integer, related with the exact value of $\theta$. Our approach relies on a spin chain regularization, where the boundary conditions now correspond to the introduction of additional edge states. The exact values of the exponents then follow from a lengthy algebraic analysis, a reformulation of the spin chain in terms of crossing and non-crossing loops (represented as a certain subalgebra of the Brauer algebra), and earlier results on the so-called one- and two-boundary Temperley Lieb algebras (also known as blob algebras). A remarkable result is that the exponents, in general, turn out to be irrational. The case $M=1$ has direct applications to the spin quantum Hall effect, which will be discussed in a sequel.
\end{abstract}




\section{Introduction}

Two-dimensional sigma models on supergroups and supercosets play a fundamental role in several areas of theoretical physics, such as phase transitions in $2+1$ non interacting disordered electronic systems $[1,2,3]$ (for reviews see $[4,5]$ ), or the AdS/CFT duality $[6,7,8,9]$. The study of these models is on the other hand quite difficult, for a variety of reasons all related to the non unitarity of the target. Progress has thus been rather slow, but has picked up pace recently, thanks to the use of mini superspace technology [10], a better understanding of algebraic aspects [11, 12, 13, 14], and the introduction of lattice regularizations $[15,16,17,18,19,20,21]$ inspired from network models $[22,23,24]$.

A particularly interesting question for critical super sigma models concerns the classification of their conformal boundary conditions. While for Wess Zumino Witten models the question is well understood [25, 26], the situation for other models - such as the superprojective sigma models at $\theta=\pi$-is much less under control. In a recent paper [16], the sigma models on

$$
\mathbb{C P}^{N-1 \mid N}=\mathrm{U}(N \mid N) /(\mathrm{U}(1) \times \mathrm{U}(N-1 \mid N))
$$

at $\theta=\pi$ were considered, and the set of conformal boundary conditions invariant under the full group $\mathrm{PU}(N \mid N)=\mathrm{U}(N \mid N) / \mathrm{U}(1)$ classified. These boundary conditions are parametrized by an integer, and correspond, in string theory parlance, to volume filling branes equipped with a monopole line bundle and a connection. In sigma model language, the existence of these boundary conditions has to do with the fact that, while the bulk theory only depends on the value of $\theta$ modulo $2 \pi$, when there is a boundary, the exact value of the topological angle matters, and enters, for instance, the classical equations of motion [27, 28, 29].

The sigma model considered in [16] is peculiar because it is critical for a large domain of values of the coupling constant $g_{\sigma}^{2}$ including the minisuperspace limit $g_{\sigma}^{2} \rightarrow 0$. The solution using harmonic analysis on the target in this limit is a precious help in finding out the exact spectrum for all values of $g_{\sigma}^{2}$ up to the critical value $g_{\sigma}^{2}=1$ (in normalizations of [16]) beyond which the sigma model exhibits different behavior. For other types of superprojective sigma models, such a line of fixed points is not available, and one must directly solve the model at finite coupling. The lattice regularizations of [17] constitute a powerful means of obtaining such a solution, and we shall take this route in the present paper.

The main objective of this work is to extend the $\mathbb{C P}^{N-1 \mid N}$ study of [16] to the considerably more difficult and richer case of $\mathbb{C P}^{N+M-1 \mid N}$. The use of lattice regularizations in this context is not only a practical tool, but also provides a physical intuition for understanding conformal boundary conditions in terms of edge states. ${ }^{1}$ This in turn has applications to the description of the transition

\footnotetext{
${ }^{1}$ We note that the role of edge states in sigma models has recently been revisited in [30], although the relation with our and other's work is not clear to us.
} 
between plateaus in the spin quantum Hall effect [31], which corresponds to the $M=1$ case of the general construction. We will discuss the implications of this observation in a subsequent work [32].

Our paper is organized as follows. In section 2, we revisit some of the main results in [16] and discuss boundary conditions in sigma models. We review in detail the easy case of $M=0$, $N=1$ (symplectic fermions) which will serve as a benchmark for the subsequent developments. In section 3, we discuss briefly the role of boundaries in mapping spin chains to sigma models, together with the concept of edge states. In section 4, we discuss the spin chains relevant for the solution of the boundary superprojective sigma models, together with their geometrical formulation in terms of loops and in terms of lattice algebras. Section 5 is the most technical: this is where we obtain the solution of the loop model based on earlier results on one and two boundary TemperleyLieb algebra, together with exact solutions for $\mathbb{C P}^{N-1 \mid N}$ and exact diagonalizations of finite size systems otherwise. In section 6 , we discuss in details the relation between the spectrum of the spin chains and loop model, while section 7 contains conclusions. Some particularly technical results are discussed in the appendices.

\section{$1.1 \quad$ Notations}

For the reader's convenience we collect here some notations to be used in this paper.

- $N$ and $N+M$ denote respectively the number of bosonic and fermionic coordinates of the superspaces used.

- $L$ is the number of pairs of alternating representations in the bulk of the spin chain.

- $m$ is the number of additional representations on the left boundary of the spin chain.

- $n$ is the number of additional representations on the right boundary of the spin chain.

- $\mathfrak{m}=\min (m, n)$ is the minimal number of uncontractible pairs of boundary representations.

- $\mathcal{L}$ and $\mathcal{R}$ are integer (monopole) numbers labelling different boundary conditions at the ends of the world-sheet in the sigma model formulation.

- $\beta$ is the fugacity of bulk loops in the geometrical formulation of the model.

- $\beta_{1}$ and $\beta_{2}$ are the fugacities of loops touching respectively the left and the right boundary in the two-boundary loop model, and $\beta_{12}$ is the fugacity of loops touching both.

- $\mathcal{B}_{2 L}$ is the Brauer algebra on $2 L$ strands.

- $\mathcal{A}_{2 L, m, n}$ is the subalgebra of the Brauer algebra involved in the formulation of the lattice model. 
- $j$ is the number of non contracted pairs of bulk lines.

- $k$ is the number of non contracted pairs of boundary lines.

- $p$ is the minimal model index, corresponding to setting $\beta=2 \cos \left(\frac{\pi}{p+1}\right)$.

- $h_{r, s}$ is the Kac table of conformal weights.

\section{Boundary sigma models}

We first briefly review the definition of the $\mathbb{C P}^{N+M-1 \mid N}$ models, and then move on to describe the role of boundaries in the sigma models.

\subsection{Superprojective sigma models at $\theta=\pi$}

Complex projective superspaces $\mathbb{C P}^{N+M-1 \mid N}$ are built much like their bosonic cousins $[17,16]$. Begin with superspace $\mathbb{C}^{N+M \mid N}$; the $N+M$ complex bosonic coordinates are denoted by $z_{a}$ and we use $\xi_{a}$ for the $N$ fermionic directions. Within this complex superspace, consider the odd (real) dimensional supersphere defined by the equation

$$
\sum_{a=1}^{N+M} z_{a} z_{a}^{*}+\sum_{a=1}^{N} \xi_{a} \xi_{a}^{*}=1 .
$$

The supersphere $S^{2 N+2 M-1 \mid 2 N}$ carries an action of $U(1)$ by simultaneous phase rotations of all bosonic and fermionic coordinates,

$$
z_{a} \longrightarrow e^{i \varpi} z_{a} \quad, \quad \xi_{a} \longrightarrow e^{i \varpi} \xi_{a}
$$

Note that this transformation indeed leaves the constraint invariant. The complex projective superspace $\mathbb{C P}^{N+M-1 \mid N}$ is the quotient space $S^{2 N+2 M-1 \mid 2 N} / U(1)$.

Functions on the supersphere $S^{2 N+2 M-1 \mid 2 N}$ carry an action of the Lie supergroup $\mathrm{U}(N+M \mid N)$. These transformations include the phase rotations (3) which act trivially on $\mathbb{C P}^{N+M-1 \mid N}$. Hence, the stabilizer subalgebra of a point on the projective superspace is given by $\mathrm{u}(1) \times \mathrm{u}(N+M-1 \mid N)$ where the first factor corresponds to the action (3). We conclude that

$$
\mathbb{C P}^{N+M-1 \mid N}=\mathrm{U}(N+M \mid N) /(\mathrm{U}(1) \times \mathrm{U}(N+M-1 \mid N)) .
$$

Their simplest representative of interest to us is $\mathbb{C P}^{0 \mid 1}$ i.e. the space with just two real fermionic coordinates. The sigma model with this target space is equivalent to the theory of two symplectic fermions, which has been extensively investigated, as for example in [33, 34].

The construction of the sigma model on $\mathbb{C P}^{N+M-1 \mid N}$ closely parallels this geometric construction. The model involves a field multiplet $Z_{\alpha}=Z_{\alpha}(z, \bar{z})$ with $N+M$ bosonic components 
$Z_{\alpha}=z_{\alpha}, \alpha=1, \ldots, N+M$ and $N$ fermionic fields $Z_{\alpha}=\xi_{\alpha-N-M}, \alpha=N+M+1, \ldots, 2 N+M+1$. To distinguish between bosons and fermions we introduce from now on a grading function $|\cdot|$, which is 0 when evaluated on the labels of bosonic and 1 on the labels of fermionic quantities. In addition we also need a non-dynamical U(1) gauge field $a$. With this field content, the action takes the form (with a summation convention on the index $\alpha$ )

$$
S=\frac{1}{2 g_{\sigma}^{2}} \int \mathrm{d}^{2} z\left(\partial_{\mu}-i a_{\mu}\right) Z_{\alpha}^{\dagger}\left(\partial_{\mu}+i a_{\mu}\right) Z_{\alpha}-\frac{i \theta}{2 \pi} \int \mathrm{d}^{2} z \epsilon^{\mu \nu} \partial_{\mu} a_{\nu}
$$

and the fields $Z_{\alpha}$ are subject to the constraint $Z_{\alpha}^{\dagger} Z_{\alpha}=1 .^{2}$ The integration over the abelian gauge field can be performed explicitly and it leads to the replacement

$$
a_{\mu}=\frac{i}{2}\left[Z_{\alpha}^{\dagger} \partial_{\mu} Z_{\alpha}-\left(\partial_{\mu} Z_{\alpha}^{\dagger}\right) Z_{\alpha}\right]
$$

The term multiplied by $\theta$ does not contribute to the equations of motion for $a_{\mu}$. As its bosonic counterpart, the $\mathbb{C P}^{N+M-1 \mid N}$ sigma model on a closed surface possesses instanton solutions. The corresponding instanton number is computed by the term - which we will denote by $Q$ and refer to as the 'topological term'- that multiplies the parameter $\theta$. Since $Q$ is integer-valued, the parameter $\theta=\theta+2 \pi$ can be considered periodic as long as the world-sheet has no boundary.

The target supermanifold being a symmetric superspace, the metric on the target space is unique up to a constant factor, so $g_{\sigma}^{2}$ and $\theta$ are the only coupling constants. The perturbative beta function is the same as the one for $\mathbb{C P}^{M-1}[35]$

$$
\frac{\mathrm{d} g_{\sigma}^{2}}{\mathrm{~d} l}=\beta\left(g_{\sigma}^{2}\right)=M g_{\sigma}^{4}+O\left(g_{\sigma}^{6}\right)
$$

The beta function for $\theta$ is zero in perturbation theory, and that for $g_{\sigma}^{2}$ is independent of $\theta$.

For $M>0$, the coupling is weak at short length scales, but flows to strong values at large length scales. For $\theta \neq \pi(\bmod 2 \pi)$ the $\mathrm{U}(N+M \mid N)$ symmetry is eventually restored, and the theory is massive. When $\theta=\pi(\bmod 2 \pi)$ and $M \leq 2$, the model flows to a non-trivial fixed point. The corresponding bulk conformal field theory has been studied in [17, 19], and presents many fascinating features. The main purpose of this paper is to study its boundary properties in more details.

For $M=2$ and $N=0$ we recover the usual flow in the $O(3)$ sigma model; the conformal field theory in that case is the $S U(2)$ level 1 Wess Zumino model. For $M=0$, the beta function is in fact exactly zero, and the sigma model exhibits a line of fixed points [17, 16, 36, 4, 37]. The case $M=1$ is particularly interesting, because it is related with percolation, and with the spin quantum Hall effect [31, 32].

\footnotetext{
${ }^{2}$ Note that we eliminated the radius $\rho$ of the complex projective space in favor of a coupling $g_{\sigma}^{-2}$ entering the action in front of the metric. Equivalently, we can set $g_{\sigma}^{2}=1$ and work with a radius parameter $\rho$ appearing in the modified constraint $Z_{\alpha}^{\dagger} Z_{\alpha}=4 \rho^{2}$.
} 


\subsection{The role of the boundaries}

While the model on a compact oriented manifold exhibits properties that do not depend on the exact value of $\theta$ provided $\theta=\pi(\bmod 2 \pi)$, things are bound to be quite different in the presence of a boundary, as first noticed in [29]. Strictly speaking, in a finite system with boundaries, there are no well defined topologically distinct sectors. One does not expect the behavior of the RG for the bulk properties to change - the standard analysis should still apply provided the equivalent of the instantons (configurations such that the 'topological term' $Q$ approaches a constant in the core, far from the boundary) are well localized within the system. But boundary properties are expected to now depend on the exact value of $\theta$; in particular, $\theta$ can now be expected to affect perturbation theory.

To make things more concrete, we restrict to sigma models on the strip $\Sigma=[0, \pi] \times \mathbb{R}$ or, equivalently by conformal transformation, the upper half plane $z=x+i y, y>0$. The boundary conditions induced by the 'topological term' are

$$
\begin{aligned}
& \left(\partial_{y}+i a_{y}\right) Z_{\alpha}=\frac{\theta}{\pi} g_{\sigma}^{2}\left(\partial_{x}+i a_{x}\right) Z_{\alpha}, \\
& \left(\partial_{y}-i a_{y}\right) Z_{\alpha}^{\dagger}=-\frac{\theta}{\pi} g_{\sigma}^{2}\left(\partial_{x}-i a_{x}\right) Z_{\alpha}^{\dagger}
\end{aligned}
$$

for $z=\bar{z}<0$ and a similar condition along the right half $z=\bar{z}>0$ of the boundary. While in the weak coupling limit the value of $\theta$ is irrelevant (and we recover purely Neumann boundary conditions), in general, $\theta$ appears explicitly.

Of course, except in the special case $M=0$ [17], we will need $\theta=\pi(\bmod 2 \pi)$ to have a conformal field theory in the low energy limit, but we can now expect properties to depend on the exact value of $\theta$ itself.

It was indeed argued in [16] that a more general family of $\mathrm{U}(N+M \mid N)$ symmetric boundary conditions can be obtained which are expressed through the conditions

$$
\begin{aligned}
& \left(\partial_{y}+i a_{y}\right) Z_{\alpha}=\Theta_{1} g_{\sigma}^{2}\left(\partial_{x}+i a_{x}\right) Z_{\alpha}, \\
& \left(\partial_{y}-i a_{y}\right) Z_{\alpha}^{\dagger}=-\Theta_{1} g_{\sigma}^{2}\left(\partial_{x}-i a_{x}\right) Z_{\alpha}^{\dagger}
\end{aligned}
$$

for $z=\bar{z}<0$ and a similar condition with $\Theta_{1}$ replaced by $\Theta_{2}$, along the right half $z=\bar{z}>0$ of the boundary. The parameters $\Theta_{1}=2 \mathcal{L}+\theta / \pi$ and $\Theta_{2}=2 \mathcal{R}+\theta / \pi$ involve now integer (monopole) numbers $\mathcal{L}, \mathcal{R}$.

In the case $M=0$, it was shown in [16] how, to every choice of integer $\mathcal{R}=\mathcal{L}$, is associated a conformally invariant boundary condition. The case $\mathcal{R} \neq \mathcal{L}$ then corresponds to the insertion of a boundary condition changing operator.

For $0<M \leq 2$, we expect similarly that conformally invariant boundary conditions at the fixed point theory are induced by these boundary terms. In other words, we expect that, in the 
conformally invariant fixed points of superprojective sigma models $\mathbb{C P}^{N+M-1 \mid N}$ at $\theta=\pi$, there is a discrete family of conformal boundary conditions labelled by the integer $\mathcal{R} \in \mathbb{Z}$ : these are the subject of our study.

We note that the only dependence on the exact value of $\theta$ comes from the boundary conditions. A model with a given value of $\theta, \mathcal{L}, \mathcal{R}$ is identical with the model with $\theta+2 p \pi, \mathcal{L}-p, \mathcal{R}-p$, for any $p \in \mathbb{Z}$.

\subsection{The case $\mathbb{C} \mathbb{P}^{0 \mid 1}$}

To make things more concrete, we note that the simplest case of $\mathbb{C P}^{0 \mid 1}(N=1, M=0)$ is equivalent to symplectic fermions. With the bulk action

$$
S=\frac{1}{2 g_{\sigma}^{2}} \int d^{2} z \partial_{\mu} \xi \partial_{\mu} \xi^{\dagger}
$$

the boundary conditions are then of the form

$$
\begin{aligned}
\partial_{y} \xi & =\Theta g_{\sigma}^{2} \partial_{x} \xi \\
\partial_{y} \xi^{\dagger} & =-\Theta g_{\sigma}^{2} \partial_{x} \xi^{\dagger}
\end{aligned}
$$

They can be represented in terms of a glueing automorphism

$$
\left(\begin{array}{c}
\partial \xi \\
\partial \xi^{\dagger}
\end{array}\right)=\Omega\left(\begin{array}{c}
\bar{\partial} \xi \\
\bar{\partial} \xi^{\dagger}
\end{array}\right)
$$

where

$$
\Omega=\frac{1+W(\Theta)}{1-W(\Theta)}, \quad W(\Theta)=i g_{\sigma}^{2}\left(\begin{array}{cc}
\Theta & 0 \\
0 & -\Theta
\end{array}\right)
$$

If we now have two different values of $\Theta$ on the left and right boundaries, going around the insertion of the boundary condition changing operator in the complex plane gives rise to a monodromy expressed by

$$
\Omega_{12}=\Omega_{1} \Omega_{2}^{-1}=\frac{\kappa+W\left(\Theta_{1}-\Theta_{2}\right)}{\kappa-W\left(\Theta_{1}-\Theta_{2}\right)}
$$

where we have set $\kappa=1+g_{\sigma}^{4} \Theta_{1} \Theta_{2}$. Of course, $\Omega_{12}$ is of the form

$$
\Omega_{12}=\left(\begin{array}{cc}
e^{2 i \pi \lambda} & 0 \\
0 & e^{-2 i \pi \lambda}
\end{array}\right)
$$

with the twist parameter $\lambda$ given by $2 \cos 2 \pi \lambda=\operatorname{Tr} \Omega_{12}$ :

$$
\cos 2 \pi \lambda=\frac{\left(1+g_{\sigma}^{4} \Theta_{1} \Theta_{2}\right)^{2}-\left(\Theta_{1}-\Theta_{2}\right)^{2} g_{\sigma}^{4}}{\left(1+g_{\sigma}^{4} \Theta_{1} \Theta_{2}\right)^{2}+\left(\Theta_{1}-\Theta_{2}\right)^{2} g_{\sigma}^{4}}
$$

The twist parameter vanishes when $\mathcal{L}=\mathcal{R}$. In general, the ground state of the theory (which has central charge $c=-2$ ) scales with the conformal weight

$$
h_{\lambda}^{g r}=\frac{1}{2} \lambda(\lambda-1)
$$


and the full operator content in this sector is encoded in the $U(1) \times$ Vir character [33]

$$
\begin{aligned}
d_{\mu, \lambda} & =\operatorname{tr}\left(e^{2 i \mu J_{0}} q^{L_{0}-c / 24}\right)= \\
& =e^{-2 i \pi \mu \lambda} q^{(1-6 \lambda(1-\lambda)) / 12} \prod_{n=1}^{\infty}\left(1+e^{2 i \pi \mu} q^{n+\lambda-1}\right)\left(1+e^{-2 i \pi \mu} q^{n-\lambda}\right)
\end{aligned}
$$

or

$$
d_{\mu, \lambda}=\eta^{-1} e^{-2 i \pi \mu \lambda} \sum_{m \in Z} e^{2 i \pi \mu m} q^{\frac{1}{2}\left(m+\lambda-\frac{1}{2}\right)^{2}},
$$

where $q$ denotes the modular parameter and $\eta$ is the Dedekind eta function.

The problem is thus fully solved in this case. In this paper, we want to address the considerably more difficult case of $M \neq 0$, in particular the case $M=1$ which is relevant to the spin quantum Hall effect [31, 32].

\section{Boundary conditions in super spin chains}

\subsection{Super spin chains}

As mentioned in the introduction, our strategy to solve the boundary sigma model is to use a lattice regularization. There is a well known, profound relationship between sigma models and spin chains, going back to the earliest developments in the $O(3)$ case [38]. In fact, the conformal fixed points in the bulk $\mathbb{C P}^{N+M-1 \mid N}$ sigma models at $\theta=\pi$ have been studied in $[17,19]$ using homogeneous super spin chains that represent the strong coupling region. The mapping to the sigma models follows the well known argument in non graded [39, 40] as well as graded [41, 31] cases.

The simplest chain (which will be described in considerably more details below) is obtained by alternating the fundamental representation $V$ of $\mathfrak{s l}(N+M \mid N)$ and its conjugate $V^{*}$, and choosing an antiferromagnetic Heisenberg coupling between nearest neighbours (since the product $V \otimes V^{*}$ decomposes generically on two representations - the identity and the adjoint - this is the most general nearest neighbour interaction). This coupling in turn can be conveniently recognized as a representation $E^{\mathfrak{s l}}$ of the Temperley-Lieb algebra acting on $\left(V \otimes V^{*}\right)^{\otimes L}$, and the Hamiltonian with open (free) boundary conditions can be written as

$$
H=-\sum_{i=1}^{2 L-1} E_{i}^{\mathfrak{s l}}
$$

Detailed expressions in terms of the natural vector basis of $V, V^{*}$ are given in $[17,18]$ and we do not reproduce them for now (but see below). An important point for further study is that the 
generators $E_{i}^{\mathfrak{s l}}$ satisfy the Temperley-Lieb relations [42]

$$
\begin{aligned}
\left(E_{i}^{\mathfrak{s l}}\right)^{2} & =M E_{i}^{\mathfrak{s l}} \\
E_{i}^{\mathfrak{s l}} E_{i \pm 1}^{\mathfrak{s l}} E_{i}^{\mathfrak{s l}} & =E_{i}^{\mathfrak{s l}} \\
{\left[E_{i}^{\mathfrak{s l}}, E_{j}^{\mathfrak{s l}}\right] } & =0, \quad \text { for }|i-j| \geq 2 .
\end{aligned}
$$

Instead of the Hamiltonian, it is sometimes useful to use a transfer matrix, the evolution operator of the $1 \mathrm{D}$ chain in discrete imaginary time. The choice of alternating representations in the spin chain corresponds then to having oriented edges carrying respectively $V$ and $V^{*}$ as illustrated in figure 1, making the potential relationship with network models of quantum localisation more transparent. The transfer matrix itself is then built as a product of two diagonal-to-diagonal transfer matrices associated to two consecutive layers of the lattice, which evolves the states one unit in time.

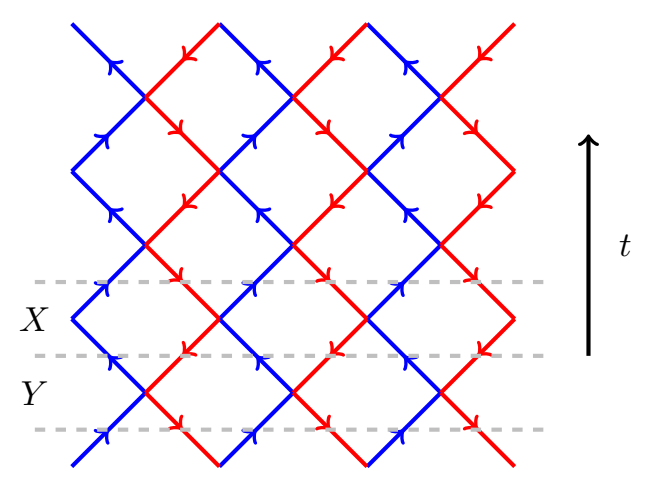

Figure 1: The two dimensional lattice corresponding to the alternating super spin chain. Edges carrying the fundamental representation have up arrows while those with the dual down arrows. The transfer matrix is a product of two transfer matrices acting on two consecutive layers $T=X Y$, imaginary time flows from bottom to top.

For $0<M \leq 2$, the model thus defined flows to the fixed points discussed in [19]. The Hamiltonian with periodic boundary conditions has been studied in [17]. When $M=0$, recall $[17,16,36,4,37]$ that the sigma model admits a line of fixed points parametrized by $g_{\sigma}^{2}$ : the model we have just defined corresponds to a particular value of $g_{\sigma}^{2}(=1)$. Other values can be obtained by adding an exactly marginal interaction corresponding to the exchange of next nearest neighbors degrees of freedom $V$ or $V^{*}$ (since the products $V \otimes V$ or $V^{*} \otimes V^{*}$ decompose generically on two representations, this is the most generic nearest neighbor coupling). In the Hamiltonian language, this means

$$
H=-\sum_{i=1}^{2 L-1} E_{i}^{\mathfrak{s l}}-w \sum_{i=1}^{2 L-2} P_{i, i+2}^{\mathfrak{s l}}
$$

where $P_{i, i+2}^{\mathfrak{s l}}$ is the operator permuting states in sites $i$ and $i+2$. Note that the $P_{i, i+2}^{\mathfrak{s l}}$ terms do not break the $\mathfrak{s l}(N+M \mid N)$ symmetry [36], i.e., it does not mix up the $V$ and $V^{*}$ spaces. A proposal 
for obtaining the conformal boundary conditions starting from this spin chain was then made in [16]. There, a family of four possible chains was considered, corresponding to the following spaces (with factors labelled from 0 to $2 L+m+n-1$ ) and Hamiltonians

$$
\begin{aligned}
& V^{\otimes m} \otimes\left(V \otimes V^{\star}\right)^{\otimes L} \otimes\left(V^{\star}\right)^{\otimes n}: \quad H^{V V^{\star}}=H_{\text {left }}^{V}+H_{\text {bulk }}+H_{\text {right }}^{V^{\star}} \\
& V^{\otimes m} \otimes\left(V \otimes V^{\star}\right)^{\otimes L} \otimes V^{\otimes n}: \quad \quad H^{V V}=H_{\text {left }}^{V}+H_{\text {bulk }}+H_{\text {right }}^{V} \\
& \left(V^{\star}\right)^{\otimes m} \otimes\left(V \otimes V^{\star}\right)^{\otimes L} \otimes\left(V^{\star}\right)^{\otimes n}: \quad H^{V^{\star} V^{\star}}=H_{\text {left }}^{V^{\star}}+H_{\text {bulk }}+H_{\text {right }}^{V^{\star}} \\
& \left(V^{\star}\right)^{\otimes m} \otimes\left(V \otimes V^{\star}\right)^{\otimes L} \otimes V^{\otimes n}: \quad \quad H^{V^{\star} V}=H_{\text {left }}^{V^{\star}}+H_{\text {bulk }}+H_{\text {right }}^{V},
\end{aligned}
$$

where the bulk Hamiltonian is

$$
H_{\mathrm{bulk}}=-\sum_{i=m}^{2 L+m-2} E_{i}^{\mathfrak{s l}}-w \sum_{i=m}^{2 l+m-3} P_{i, i+2}^{\mathfrak{s l}},
$$

while the boundary Hamiltonians are as follows

$$
\begin{aligned}
H_{\text {left }}^{V} & =-u \sum_{i=0}^{m-1} P_{i, i+1}^{\mathfrak{s l}} \quad H_{\text {right }}^{V^{\star}}=-v \sum_{i=2 L+m-1}^{2 L+m+n-2} P_{i, i+1}^{\mathfrak{s l} l} \\
H_{\text {left }}^{V^{\star}} & =-u \sum_{i=0}^{m-2} P_{i, i+1}^{\mathfrak{s l}}-w^{\prime} P_{m-1, m+1}^{\mathfrak{s l}}-t^{\prime} E_{m-1}^{\mathfrak{s l}} \\
H_{\text {right }}^{V} & =-t^{\prime \prime} E_{2 L+m-1}^{\mathfrak{s l}}-w^{\prime \prime} P_{2 L+m-2,2 L+m}^{\mathfrak{s l}}-v \sum_{i=2 L+m}^{2 L+m+n-2} P_{i, i+1}^{\mathfrak{s l}} .
\end{aligned}
$$

In words, the Temperley-Lieb generators operate only in the bulk spaces, whereas the permutation operators act on the boundary spaces and on the first physical space of the same type as the boundary spaces.

Numerical evidence was presented [16] to the effect that these chains correspond to the sigma model with monopole boundary conditions according to the correspondence

$$
\begin{array}{rlrl}
V^{\otimes m} \otimes\left(V \otimes V^{\star}\right)^{\otimes L} \otimes\left(V^{\star}\right)^{\otimes n}: & \mathcal{L}=+m & \mathcal{R}=+n, \\
V^{\otimes m} \otimes\left(V \otimes V^{\star}\right)^{\otimes L} \otimes V^{\otimes n}: & \mathcal{L}=+m & \mathcal{R}=-n, \\
\left(V^{\star}\right)^{\otimes m} \otimes\left(V \otimes V^{\star}\right)^{\otimes L} \otimes\left(V^{\star}\right)^{\otimes n}: & \mathcal{L}=-m & \mathcal{R}=+n, \\
\left(V^{\star}\right)^{\otimes m} \otimes\left(V \otimes V^{\star}\right)^{\otimes L} \otimes V^{\otimes n}: & \mathcal{L}=-m & \mathcal{R}=-n .
\end{array}
$$

It was also argued that

$$
\theta=\pi
$$

and that, for the value $w=0$ to which we restrict here,

$$
M=0, \quad g_{\sigma}^{2}=1 .
$$




\subsection{Mapping on sigma models: the role of boundaries}

We can give a quick heuristic derivation of these results following [43, 44]. Consider the standard mapping of the spin chain onto the sigma model. Calling the elementary Berry phase $\Omega(i)$, the total phase for the usual antiferromagnetic chain of spin $s$ would read

$$
\Phi_{\mathrm{top}}=s \sum_{i=1}^{2 L}(-1)^{i} \Omega(i) \approx \frac{s}{2} \int_{0}^{2 L} \frac{\partial \Omega}{\partial x} d x=\frac{s}{2}[\Omega(2 L)-\Omega(0)+4 \pi Q]
$$

where $Q$ is an integer. Let us now suppose we have for instance the first chain in equation (28). Since on the boundary we have representations of the same kind, the Berry phase on that side comes unstaggered, so we have (from now on we put $s=\frac{1}{2}$, which is the right value for our spin chain [17])

$$
\Phi_{\text {top }}=-\frac{m}{2} \Omega(0)+\frac{n}{2} \Omega(2 L)+\frac{1}{2} \sum_{i=1}^{2 L}(-1)^{i} \Omega(i) \approx \frac{n+1 / 2}{2} \Omega(2 L)-\frac{m+1 / 2}{2} \Omega(0)+\pi Q
$$

giving immediately rise to the equations (9) with $\theta=\pi$. Meanwhile, say for the second chain in (28) we have

$$
\Phi_{\text {top }}=-\frac{m}{2} \Omega(0)-\frac{n}{2} \Omega(2 L)+\frac{1}{2} \sum_{i=1}^{2 L}(-1)^{i} \Omega(i) \approx \frac{-n+1 / 2}{2} \Omega(2 L)-\frac{m+1 / 2}{2} \Omega(0)+\pi Q
$$

again in agreement with equations (9) this time with $\mathcal{L}=m$ but $\mathcal{R}=-n$. Note that in this kind of argument, the exact nature of the couplings on the boundary is irrelevant. Note also that it does not make a difference whether the spins on the boundary are actually projected onto the fully symmetric representation or not: we will discuss this in more details below.

We thus see that adjusting the $\mathcal{L}, \mathcal{R}$ terms in the formal boundary action of the sigma model is equivalent to adding extra spins - or edge states - to the boundaries of the spin chain. As commented earlier, the model with $\theta=\pi$ and $\mathcal{L}=\mathcal{R}=p \neq 0$ is equivalent to the same model with $\theta=\pi+2 \pi p$ and $\mathcal{L}=\mathcal{R}=0$. Hence shifting $\theta$ by multiples of $2 \pi$ leads to the apparition of extra edge states. This phenomenon is well known in the language of QED (see [45]), to which the $\mathbb{C} P^{M-1}$ model is equivalent at large $M$, with $M$ flavors and a weak gauge coupling $e^{2} \approx \frac{1}{M}$, as reviewed in [39]. The topological term is equivalent to a background electrostatic field $F$ in the one-dimensional universe, with $F=\frac{e \theta}{2 \pi}$. If this field is too large, it is energetically favorable to produce quark-antiquark pairs. For one such pair, $l$ being the distance between the quark and antiquark, the difference in energy between the state with and without the pair is

$$
\Delta E=l\left[(F \pm e)^{2}-F^{2}\right]
$$

While it is not energetically favourable for the vacuum to produce a pair if $|F| \leq \frac{1}{2} e$, it becomes so if $|F|>\frac{1}{2} e$. Pairs will in fact be produced until $F$ is brought down to a value $\left|F_{\text {screened }}\right| \leq \frac{1}{2} e$. So 
if $\pi<\theta<3 \pi$, one pair is produced, and more generally if $(2 n-1) \pi<\theta<(2 n+1) \pi, n$ pairs are produced.

This is in fact for $F$ positive. If $F$ is negative, that is if $\theta<0$, things are quite similar, only one produces this time antiquark-quark pairs. The picture is thus in agreement with the previous findings in the case where there are extra representations $V^{p}$ on the left and $\left(V^{*}\right)^{p}$ on the right $(\mathcal{L}=\mathcal{R}=p)$, or $\left(V^{*}\right)^{p}$ on the left and $V^{p}$ on the right $(\mathcal{L}=\mathcal{R}=-p)$, with $\Theta=\frac{\theta}{\pi}+2 \mathcal{R}=1+2 \mathcal{R}$. This is once we have identified the chain with no extra representations as corresponding to $\theta=\pi$ $\left(s=\frac{1}{2}\right)$.

Restricting now to the case $\mathcal{L}=\mathcal{R}$, we observe that another way to have the same physics would be, instead of taking a spin chain with $s=\frac{1}{2}$ in the bulk and adding edge states by hand, to take

directly a chain with $\operatorname{spin} s=\frac{1}{2}+\mathcal{L}=\frac{1+2 \mathcal{L}}{2}$. In other words, with a spin $s$ ( $s$ half an odd integer) chain in the bulk, we expect physics of edge states with $\mathcal{L}=s-\frac{1}{2}$, which corresponds in turn to a spin on the boundary with value $s^{\prime}=\frac{s-1 / 2}{2}$.

In the usual case of the $\mathbb{C P}^{1}$ model - that is, the $O(3)$ model and the XXX spin chain - all this discussion is somewhat irrelevant. Although edge states still present interesting features, we anticipate that adding extra spins (maybe in a higher dimensional representation) on the boundary does not give rise to new exponents, and the spectrum will in the end coincide with that of the XXX chain with an odd or an even number of sites. In the case of $\mathbb{C P}^{N+M-1 \mid N}$ sigma models however, an infinity of conformal boundary conditions is available, leading to a much richer behavior.

\section{Spin chains and loop models}

In what follows we will derive results for super spin chains of the type described in section 3.1 when $M$ is not fixed to zero. In particular, we focus now on the case of the chain in equation (28), which we recall:

$$
V^{\otimes m} \otimes\left(V \otimes V^{\star}\right)^{\otimes L} \otimes\left(V^{\star}\right)^{\otimes n},
$$

where as before $V$ and $V^{\star}$ are two graded vector spaces of even dimension $N+M$ and odd dimension $N$, taken respectively as the fundamental and the dual representation of $\mathfrak{s l}(N+M \mid N)$.

\subsection{Hamiltonians and transfer matrices}

We give first the explicit expression of the action of operators on this spin chain. Let $e_{j}$, with $j=1, \ldots, 2 N+M$, be a basis of $V$, and $e^{j}$, with $j=1, \ldots, 2 N+M$, be the corresponding dual basis of $V^{\star}$, and let $|j|$ denote the grade of $e_{j}$. Then the graded permutation of index $i, i+1$, with $0 \leq i \leq m-1$ or $m+2 L-1 \leq i \leq m+2 L+n-2$, acts on two copies of the same representation by interchanging them with a minus sign if both the elements are odd:

$$
P_{i, i+1}^{\mathfrak{s l}} e_{j_{0}} \otimes \cdots \otimes e^{j_{m+2 L+n-1}}=(-)^{\left|j_{i}\right|\left|j_{i+1}\right|} e_{j_{0}} \otimes \cdots \otimes e_{j_{i+1}} \otimes e_{j_{i}} \otimes \cdots \otimes e^{j_{m+2 L+n-1}}
$$


The Temperley-Lieb operator of index $i, m \leq i \leq m+2 L-2$ projects onto the singlet in the decomposition of $V \otimes V^{\star}$ (and $\left.V^{\star} \otimes V\right)$ :

$$
\begin{aligned}
E_{i}^{\mathfrak{s l}} e_{j_{0}} \otimes \cdots \otimes e^{j_{m+2 L+n-1}}= & (-)^{\left|j_{i}\right|\left|j_{i+1}\right|} \delta_{i}^{i+1} \sum_{k=1}^{N+M} e_{j_{0}} \otimes \cdots \otimes e^{j_{i-1}} \otimes \\
& \otimes e_{k} \otimes e^{k} \otimes e_{j_{i+2}} \otimes \cdots \otimes e^{j_{m+2 L+n-1}}
\end{aligned}
$$

The model we consider is given by the following Hamiltonian:

$$
H=-u \sum_{i=0}^{m-1} P_{i, i+1}^{\mathfrak{s l}}-\sum_{i=m}^{2 L+m-2} E_{i}^{\mathfrak{s l}}-v \sum_{i=2 L+m-1}^{2 L+m+n-2} P_{i, i+1}^{\mathfrak{s l}} .
$$

One can naturally associate to this Hamiltonian a vertex model defined on a strip of length $m+2 L+n$, see figure 2. Like before, the orientations of edges are fixed by the presence of $V$ and $V^{\star}$, so there are four types of vertices.

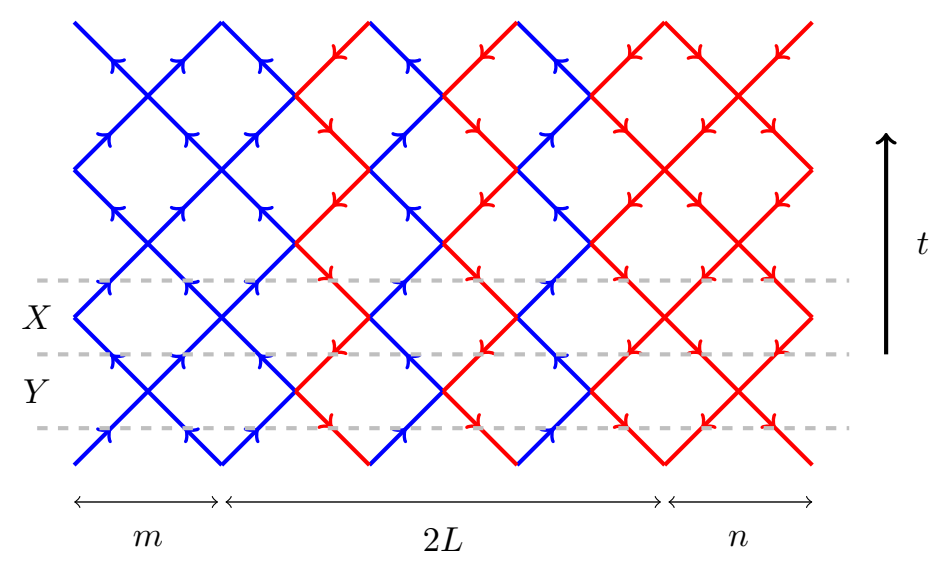

Figure 2: Vertex model corresponding to the super spin chain with extra edges at the boundaries. Here $m=n=2$.

The transfer matrix at the critical point will be of the form $T=X Y$ :

$$
\begin{aligned}
& X=\prod_{i=0}^{\mathfrak{i}^{-}(m)}\left(1+u P_{2 i+1,2 i+2}^{\mathfrak{s l}}\right) \prod_{i=\mathfrak{i}(m)}^{L-1+\mathfrak{i}^{-}(m)}\left(1+E_{2 i+1}^{\mathfrak{S l}}\right) \prod_{i=L+\mathfrak{i}^{-}(m)}^{L-1+\mathfrak{i}^{-}(m)+\mathfrak{i}^{+}(n)}\left(1+v P_{2 i+1,2 i+2}^{\mathfrak{s l}}\right) \\
& Y=\prod_{i=0}^{\mathfrak{i}^{+}(m)-1}\left(1+u P_{2 i, 2 i+1}^{\mathfrak{s l}}\right) \prod_{i=\mathfrak{i}^{+}(m)}^{L-1+\mathfrak{i}(m)}\left(1+E_{2 i}^{\mathfrak{s l}}\right) \prod_{i=L+\mathfrak{i}(m)}^{L-1+\mathfrak{i}(m)+\mathfrak{i}^{+}(n)}\left(1+v P_{2 i, 2 i+1}^{\mathfrak{s l}}\right),
\end{aligned}
$$

where $\mathfrak{i}(x)$ and $\mathfrak{i}^{ \pm}(x)$ are the integer part of $x / 2$ and $(x \pm 1) / 2$ respectively. The parameters $u, v$ are not necessarily the same as those in the Hamiltonian, but they conceal the same physics: they control the strength of the non-trivial part of the boundary interaction. A more general transfer 
matrix could be introduced with the $E_{i}^{\mathfrak{s l}}$ interaction chosen different for even and odd $i$, as is the case for the purely alternating model [17].

\subsection{Formulation within the Brauer algebra}

As mentioned earlier, the generators $E^{\mathfrak{s l}}$ obey the defining relations of the Temperley-Lieb algebra. Adding the $P^{\mathfrak{s l}}$ generators as needed to define the Hamiltonian (40), the Temperley-Lieb relations are completed by additional ones, which define a certain subalgebra of the Brauer algebra that we will denote $\mathcal{A}_{2 L, m, n}$.

It is useful at this stage to remind the reader that the full Brauer algebra on $2 L$ sites $\mathcal{B}_{2 L}(\beta)$ is generated by the permutation operators $P_{i}\left(:=P_{i, i+1}\right)$, with $i=0, \ldots, 2 L-2$, and the TemperleyLieb generators $E_{i}$, with $i=0, \ldots, 2 L-2$, and is specified by another parameter $\beta$ which we will call - for reasons that will soon become obvious - the loop fugacity $\beta$.

It is well known that words in $\mathcal{B}_{2 L}(\beta)$ can be represented graphically using diagrams composed by rows of dots connected in pairs. The generators $I$ (identity), $E_{i}$ (Temperley-Lieb operator) and $P_{i}$ (permutation operator) are shown in figure 3 in this graphical representation. The Brauer algebra is then the $\mathbb{C}$-span of diagrams thus obtained. The product of diagrams $d_{1} \cdot d_{2}$ is defined by placing $d_{1}$ over $d_{2}$ and identifying the bottom dots of $d_{1}$ with the top dots of $d_{2}$, replacing every loop formed by the (complex) weight $\beta$. For an abstract introduction to the Brauer algebra, see $[46,47]$, and for a study in the context of super spin chains, see [15].

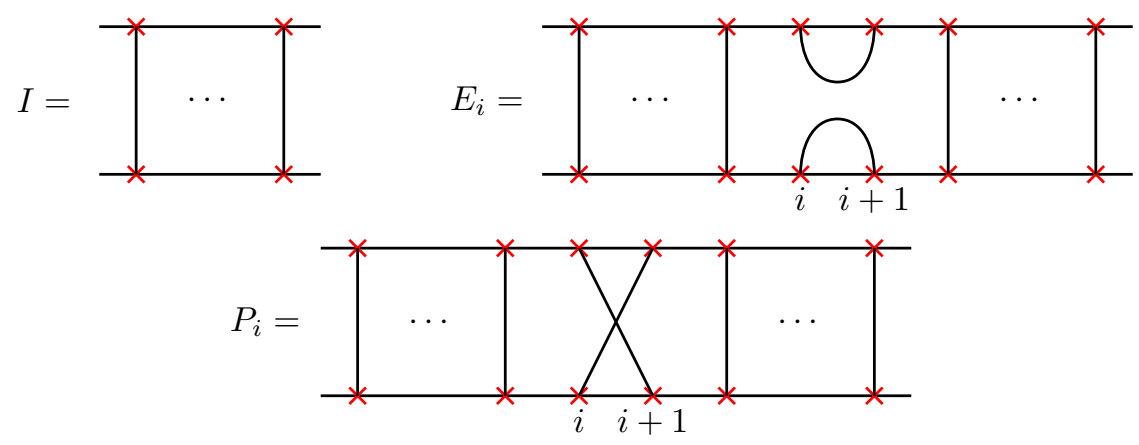

Figure 3: Identity, the Temperley-Lieb generator $E_{i}$ and the permutation operator $P_{i}$, in the diagrammatic representation.

The abstract relations defining $\mathcal{B}_{2 L}(\beta)$ are the following:

$$
\begin{aligned}
& P_{i}^{2}=1, \quad E_{i}^{2}=\beta E_{i}, \quad E_{i} P_{i}=P_{i} E_{i}=E_{i}, \\
& P_{i} P_{i \pm 1} P_{i}=P_{i \pm 1} P_{i} P_{i \pm 1}, \quad E_{i} E_{i \pm 1} E_{i}=E_{i}, \\
& P_{i} E_{i \pm 1} E_{i}=P_{i \pm 1} E_{i}, \quad E_{i} E_{i \pm 1} P_{i}=E_{i} P_{i \pm 1} .
\end{aligned}
$$


and for non-adjacent sites, $|i-j|>1$, all generators commute:

$$
P_{i} P_{j}=P_{j} P_{i}, \quad E_{i} E_{j}=E_{j} E_{i}, \quad E_{i} P_{j}=P_{j} E_{i} .
$$

In the case we wish to study, the full Brauer algebra $B_{2 L}(\beta)$ is not needed, since we restrict to having permutations on boundary sites only (the Temperley-Lieb generators still act in the bulk). The corresponding subalgebra will be denoted $\mathcal{A}_{2 L, m, n}(\beta)$, and its generators satisfy the relations

$$
\begin{aligned}
& P_{i}^{2}=1, \quad E_{i}^{2}=\beta E_{i}, \quad E_{i} P_{i}=P_{i} E_{i}=E_{i}, \\
& P_{i} P_{i \pm 1} P_{i}=P_{i \pm 1} P_{i} P_{i \pm 1}, \quad E_{i} E_{i \pm 1} E_{i}=E_{i}, \\
& E_{i} P_{i \pm 1} E_{i}=E_{i}, \\
& P_{i} P_{j}=P_{j} P_{i}, \quad E_{i} E_{j}=E_{j} E_{i}, \quad E_{i} P_{j}=P_{j} E_{i},
\end{aligned}
$$

where we note that the two relations (44) have been replaced by the single relation (48) (which is implied by the former two within the full Brauer algebra). One can easily verify these defining relations by expressing the generators as diagrams.

We shall not devote much time to studying the algebra $\mathcal{A}_{2 L, m, n}(\beta)$ abstractly, as our only purpose is to use it as a tool to diagonalize our Hamiltonians. Apart from providing a convenient language and intuition, the algebraic point of view indeed will allow us to separate the spectrum into different sectors [48], and, within each sector, to considerably reduce the size of the Hilbert space necessary for numerical determination or verification of the exponents.

\subsection{Spin chain representation and quotients}

In the spin chain, a representation of the algebra $\mathcal{A}_{2 L, m, n}(\beta)$, with loop fugacity $\beta$ equal to $\mathrm{STr} 1=$ $M$, is obtained if we take the action of $P_{i}$ and $E_{i}$ given by $P_{i}^{\text {sl }}$ and $E_{i}^{\mathfrak{s l} l}$ (see eqs. (38) and (39)). This representation is however not faithful: in addition to (46)-(49), the generators acting on the spin chain satisfy additional relations. These additional relations define a quotient of $\mathcal{A}_{2 L, m, n}(M)$, which we now describe.

First we note that the algebra $\mathcal{A}_{2 L, m, n}(M)$ as defined by (46)-(49) is infinite dimensional. We can understand why by considering the simple system with $L=m=n=1$. If we call $W=E_{1} P_{2} P_{0} E_{1}$, then the word $W^{p}$ (see figure 4) cannot be reduced using the defining relations, and increasing the power $p$, the dimension of the algebra becomes arbitrarily large.

It is also clear that this feature is present only if both $m$ and $n$ are non zero, and the larger $m$ and $n$, the larger the number of words that cannot be reduced. A similar situation appears also for the two-boundary Temperley-Lieb algebra [49], which indeed is related to our model as will be explained later.

The proper algebraic setting for our model is a quotient of $\mathcal{A}_{2 L, m, n}(M)$. We will now give the expression for the additional relations present in the spin chain representation. Call $\mathfrak{m}=\min (m, n)$ 

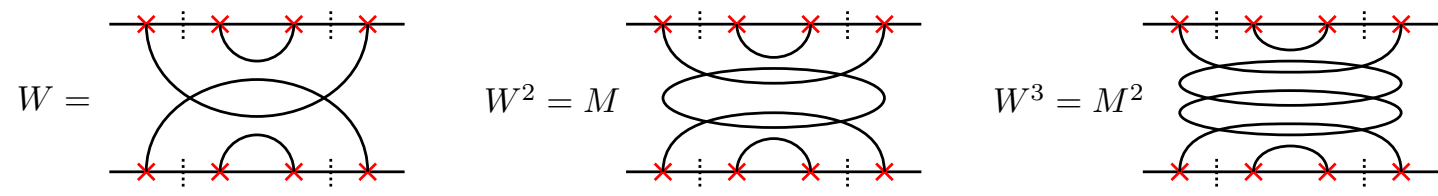

Figure 4: The diagrams corresponding to the words $W=E_{1} P_{2} P_{0} E_{1}, W^{2}$ and $W^{3}$ in $\mathcal{A}_{2 L, m, n}(M)$ with $L=m=n=1$. Here and in the following dotted lines separate bulk and boundary sites.

and

$$
E=\prod_{i \in I_{1}} E_{i} \prod_{i \in I_{2}} E_{i}
$$

where $I_{1}\left(I_{2}\right)$ is the set of even (odd) indices of Temperley-Lieb generators if $m$ is odd or the set of odd (even) indices of Temperley-Lieb generators if $m$ is even. To proceed, let us define the words $W_{r}$ by the recurrence relations:

$$
\begin{aligned}
& W_{0}=E \\
& W_{r}=E P_{m-1} \cdots P_{m-r} P_{m+2 L-1} \cdots P_{m+2 L+r-2} W_{r-1},
\end{aligned}
$$

where $r=1, \ldots, \mathfrak{m}$. Also define

$$
K_{i r}=P_{m-i} W_{r}-P_{m+2 L+i-2} W_{r}, \quad i=1, \ldots, r,
$$

for $r=1, \ldots, \mathfrak{m}$, and call the ideal generated by these elements $I$. We can now take the quotient $\mathcal{Q}_{2 L, m, n}(M)=\mathcal{A}_{2 L, m, n}(M) / I$ : we claim that the super spin chain representation of $\mathcal{Q}_{2 L, m, n}(M)$ is faithful for a sufficiently large number of states $M$.

The mechanism underlying the relations in the quotient can be understood from simple examples and easily generalized. Take again the system $L=m=n=1$. Then the additional relation in the spin chain is $P_{0} E_{1} P_{0} P_{2} E_{1}=P_{2} E_{1} P_{0} P_{2} E_{1}$. Indeed If we compute the action of $E_{1} P_{0} P_{2} E_{1}$ on the spin chain, we have:

$$
\begin{array}{r}
E_{1} P_{0} P_{2} E_{1} e_{j_{0}} \otimes e_{j_{1}} \otimes e^{j_{2}} \otimes e^{j_{3}}=(-)^{\left|j_{1}\right|} \delta_{j_{1}}^{j_{2}} \delta_{j_{0}}^{j_{3}} \sum_{k, r=1}^{M+N}(-)^{|k|\left(\left|j_{0}\right|+\left|j_{3}\right|\right)} . \\
\cdot e_{k} \otimes e_{r} \otimes e^{r} \otimes e^{k},
\end{array}
$$

that is invariant under the action of $P_{0} P_{2}$.

It is useful to represent diagrammatically the relations. In figure 5 we have represented them for the case $L=3, m=n=2$.

Note that the additional relations just introduced correctly reduce those words that made the algebra infinite dimensional earlier.

From the operational point of view which will be adopted later for numerical diagonalization, the quotient tells us that when intertwined top arcs intersect bottom arcs, their relative intertwining does not matter, and could be disentangled. 

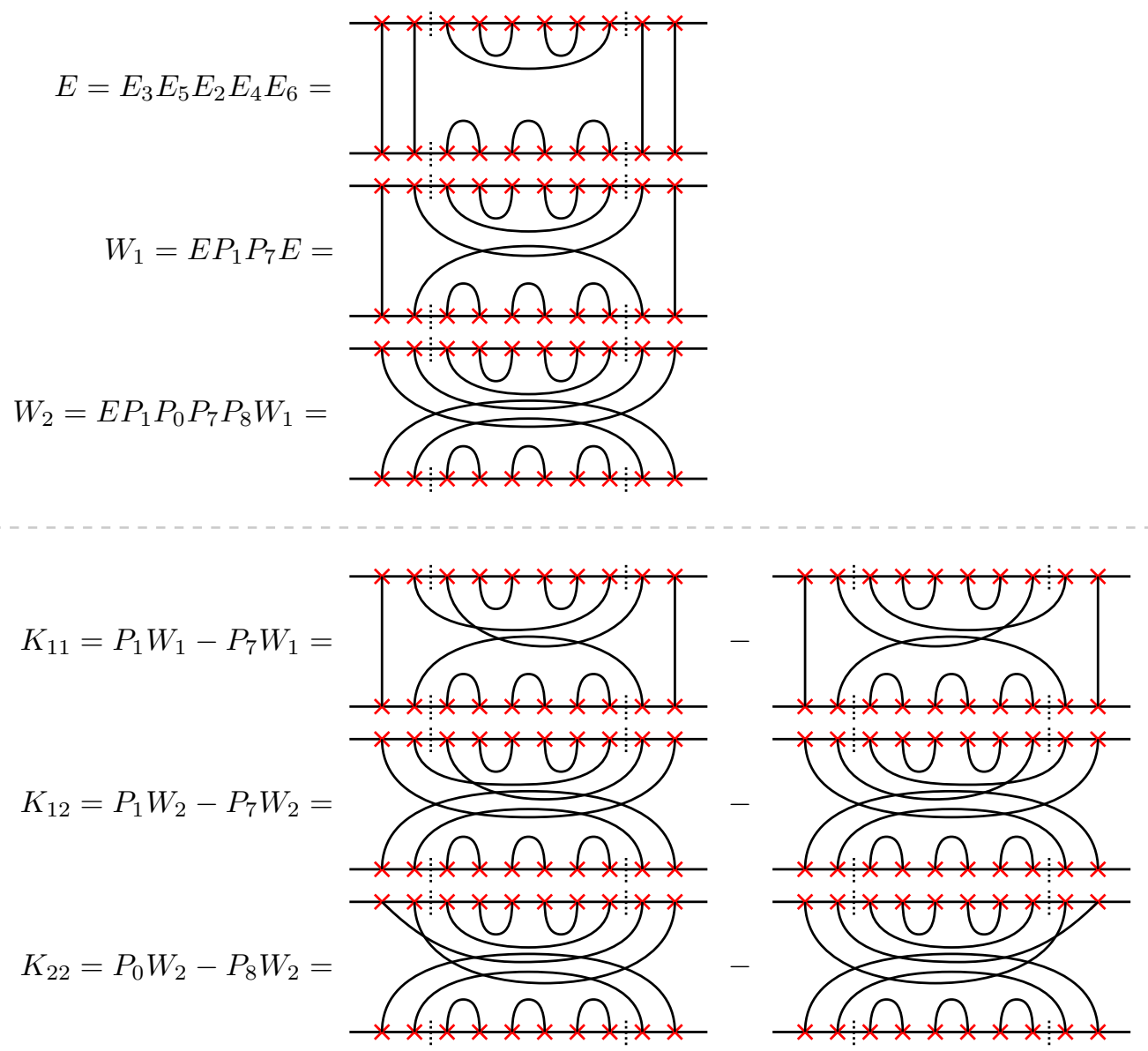

Figure 5: $K_{11}=0, K_{12}=0, K_{22}=0$ are the additional relations present in the super spin representation for a system with $L=3, m=2, n=2$. 


\subsection{Geometrical formulation: the loop model representation}

The diagram representation of the generators in our algebra naturally leads to a loop reformulation of the model: we can simply expand the product (41) in the vertex model transfer matrix and translate each term to obtain a sum over configurations of oriented loops. When viewed along the time direction, bulk vertices correspond to the junction of two incoming lines with different orientations. These can be contracted (by $E_{i}$ ) or go through (by $I$ ), as shown in the lower two lines of figure 6. On the other hand, boundary vertices correspond to incoming lines having identical orientations. These can cross (by $P_{i}$ ) or go through (by $I$ ), as shown in the upper two lines of figure 6 .

There is thus a total of four possible vertices, which can each be resolved in two ways.
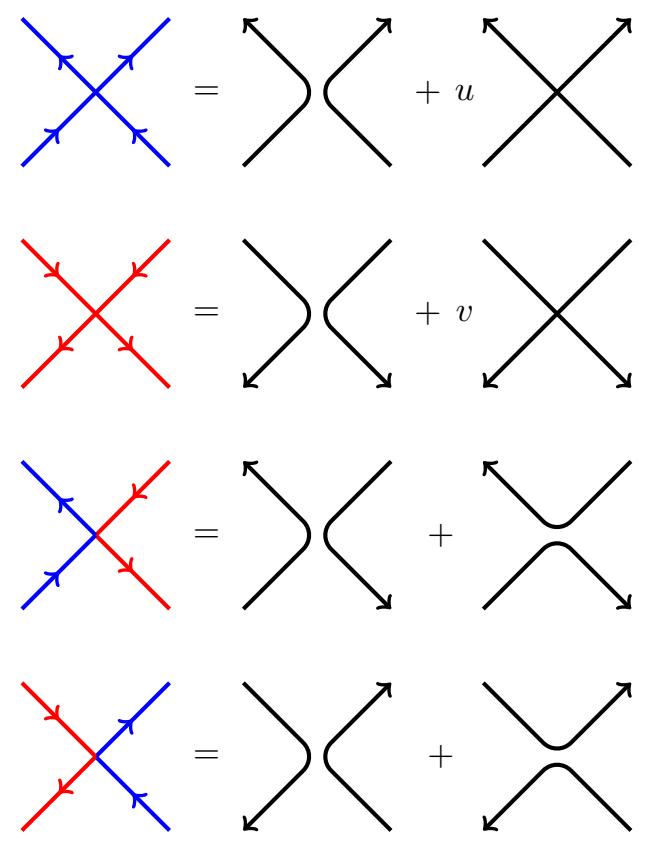

Figure 6: Vertices of the isotropic vertex model decomposed into the oriented loop configurations. $u$ and $v$ are the weights of permutations of respectively up and down links.

Expanding all the vertices into pieces of oriented loops we end up with a sum over configurations like the one illustrated in figure 7.

Note that while the diagrams used earlier to define the Brauer algebra involve unoriented lines, we have here defined the loop configurations in terms of oriented lines. The subalgebra $\mathcal{A}_{2 L, m, n}$ respects this orientation, in the sense that the associated Hamiltonian (40) and transfer matrix (41) only generate diagrams in which each loop has a definite orientation. We stress that this orientation is a property of the underlying lattice, not of the loop itself. In particular, for a loop 


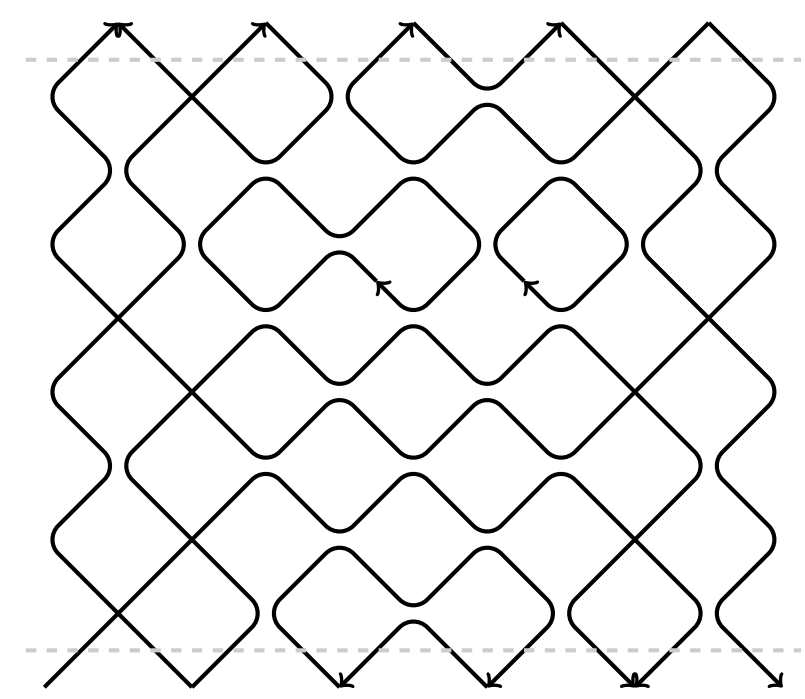

Figure 7: A configuration of the supersymmetric lattice model in the case $m=n=2,2 L=6$. The loop orientation follows the presence of the fundamental representation of the Lie superalgebra $\mathfrak{s l}(N+M \mid N)$ (links oriented up) or the dual representation (links oriented down). Each loop carries a weight $M$.

of a given position and shape, no sum over orientations is implied, and the states entering the loop representation do not contain any orientational information. The weight of the loop $\beta$ is a parameter of the algebra.

We shall find it convenient to modify the loop model in such a way that its spectrum is as close as possible to the spectrum of the spin chain. To this end we obviously set $\beta=M$. Moreover, we need to go to the quotient $\mathcal{Q}_{2 L, m, n}(M)$. As a concrete example, the word corresponding to figure 7 in the algebra $\mathcal{A}_{2 L, m, n}(M)$, will be reduced as showed in figure 8 using the additional relations of the quotient discussed above.
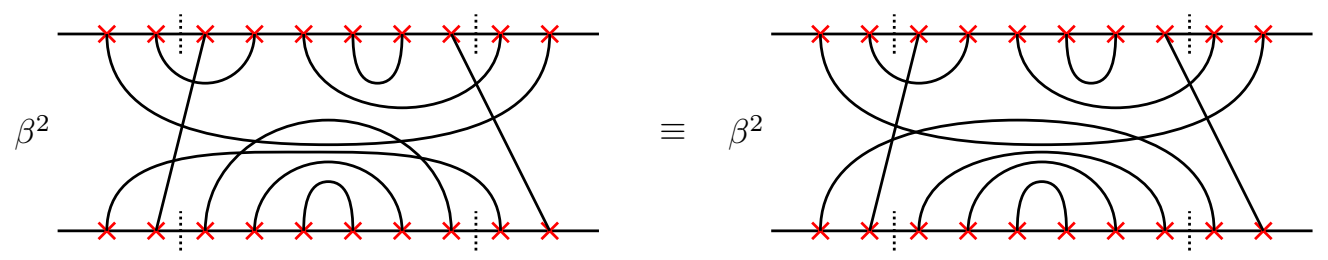

Figure 8: The two identified words in the quotient $\mathcal{Q}_{2 L, m, n}(M)$ corresponding to figure 7 .

These additional rules complete the definition of our loop model, and we can finally relate the spectrum of the Hamiltonian (40) in the loop and super spin representations. This exercise is similar to the better known cases of the Temperley-Lieb [17] and Brauer [15] algebra. For the 
Temperley-Lieb case, the spectrum of the Hamiltonian (on a given number of sites $2 L$ ) acting on the $\mathfrak{s l}(N+M \mid N)$ chain is the same for every integer $N$, the only thing that changes are (non zero) multiplicities, that grow very fast with $N$. In our case, the situation is a bit different. First, we have extensive numerical evidence that, calling $\Sigma(N)$ the set of eigenvalues of the $\mathfrak{s l}(N \mid N)$ Hamiltonian (up to non zero degeneracies), the following relations holds:

$$
\Sigma(1) \subset \Sigma(2)=\Sigma(3)=\cdots=\Sigma(N)=\ldots
$$

Moreover, $\Sigma(2)$ is also equal to the set of eigenvalues of the Hamiltonian in the loop representation: in other words, all spin chains but the $\mathfrak{s l}(1 \mid 1)$ one have the same spectrum as the geometrical model. The spectrum of $\mathfrak{s l}(1 \mid 1)$ meanwhile is only a subset; this is discussed in more details in the appendix A.

When we look at $\mathfrak{s l}(N+M \mid N), M>0$, the numerical evidence is that the spectra are all the same (again, up to non zero degeneracies). It is very likely that spin chains for a number of states per site greater or equal to 3 provide faithful representations of the quotient algebra $\mathcal{Q}_{2 L, m, n}(M)$. This statement is in line with the findings of [50], which should be possible to generalize in our case.

A final advantage of the loop representation is that we can now study the geometrical model for arbitrary values of $\beta$, which is now a simple parameter appearing as the fugacity of loops. Note that, except when $\beta=M$ is an integer, we do not know of any spin chain representation of the corresponding algebras, be they $\mathcal{A}, \mathcal{Q}$ or Brauer. This situation has to be contrasted with the ordinary Temperley-Lieb algebra, where the XXZ spin chain provides such a representation for all values of the loop fugacity. Clearly the presence of loop crossings - even if only between boundary lines - makes the problem considerably more involved. These crossings also make impossible a Coulomb gas approach to the underlying conformal field theory (for essentially the same technical reasons).

We remark that the bulk part of the Hamiltonian (40) does not follow from the usual YangBaxter construction for alternating fundamental and dual representations of $\mathfrak{s l}(N+M \mid N)$ [51]. However, in the bulk, (40) is solvable exactly, since it belongs to the Temperley-Lieb algebra, and the realization of this algebra in the $U_{q}(\mathfrak{s r}(2))$-invariant XXZ spin chain leads to Bethe ansatz equations etc (see also [52] for a related situation). We do not know what the situation for our model with boundary interactions might be. But it is interesting to observe that in the particular case of $\mathfrak{s l}(2)$, solvable spin chain Hamiltonians with boundary impurity spins can be obtained from solutions of the Yang-Baxter equation [53]. More work is needed to clarify the situation. 


\section{Critical exponents}

We now launch into the very technical problem of determining the critical exponents for the geometrical model. This will allow us later to reconstruct the spectrum of the super spin chains by the proper combination of sectors.

Our loop model in the bulk is nothing but the well known dense loop gas going back to early studies of the Potts model and the dense $O(n)$ model [54,55]. It is critical for $0 \leq M \leq 2$.

The central charge of the dense loop gas is

$$
c=1-\frac{6}{p(p+1)},
$$

if we parametrize $\beta=2 \cos \left(\frac{\pi}{p+1}\right)$. We also recall the Kac formula

$$
h_{r, s}=\frac{((p+1) r-p s)^{2}-1}{4 p(p+1)},
$$

which is often a convenient means of stating our results on critical exponents. In the following we are going to diagonalize the Hamiltonian (40), although as already stressed, we believe that the same results can be obtained studying the transfer matrix (41). Conformal invariance relates the exponents $h$ to the eigenvalues $\mathcal{E}_{i}$ of the Hamiltonian acting on a chain of length $L$ via the finite size scaling formula $[56,57]$ :

$$
\mathcal{E}_{i}=L f_{b}+2 f_{s}-\frac{v_{s} c_{\mathrm{eff}} \pi}{24 L}+\mathcal{O}\left(\frac{1}{L^{2}}\right)
$$

In this formula $f_{b}$ and $f_{s}$ are the bulk and surface free energy, $c_{\mathrm{eff}}=c-24 h$ and $v_{s}=(p+1) \sin \left(\frac{\pi}{p+1}\right)$ is the so-called velocity of sound.

We briefly review how critical exponents are related to the representation theory of the loop model (see [58] for more details). On the lattice the geometrical transfer matrix and Hamiltonians have a blockwise lower-triangular structure since the $E_{i}$ annihilate non-contractible lines (or strings) in pairs but cannot create any string and $P_{i}$ does not alter the number of strings. The restriction to a sector is done by setting the action of TL generators on two strings equal to zero. Further we note that blocks only differing by the configuration of the bottom row of states are identical. So for the purpose of computing the spectrum, we can consider reduced states in which only the top part of a state on which the Hamiltonian acts is taken. The critical exponents we are going to compute, called in the literature watermelon or $2 j$-leg exponents, are the lowest conformal weights in a sector with fixed number of non-contractible lines.

Before moving on, it is useful to recall some results about the two-boundary loop model (2BLM) $[49,59]$ which we will use later. This model is a dense loop model on a strip in which lines touching a boundary get marked in different ways, and marked loops get a weight different from the weight 
$\beta$ of bulk loops. These boundary weights are $\beta_{1}$ (resp. $\beta_{2}$ ) for loops touching the left (resp. right) boundary only, and $\beta_{12}$ for loops touching both boundaries. Using the parametrization

$$
\begin{gathered}
\beta_{1}=\frac{\sin \left(\left(r_{1}+1\right) \frac{\pi}{p+1}\right)}{\sin \left(r_{1} \frac{\pi}{p+1}\right)}, \quad \beta_{2}=\frac{\sin \left(\left(r_{2}+1\right) \frac{\pi}{p+1}\right)}{\sin \left(r_{2} \frac{\pi}{p+1}\right)} \\
\beta_{12}=\frac{\sin \left(\left(r_{1}+r_{2}+1-r_{12}\right) \frac{\pi}{2(p+1)}\right) \sin \left(\left(r_{1}+r_{2}+1+r_{12}\right) \frac{\pi}{2(p+1)}\right)}{\sin \left(r_{1} \frac{\pi}{p+1}\right) \sin \left(r_{2} \frac{\pi}{p+1}\right)},
\end{gathered}
$$

it was found that in the sector with no non-contractible lines, the conformal weights appearing are $[59]$

$$
h_{r_{12}-2 n, r_{12}},
$$

with $n \in \mathbb{Z}$, while with $2 j>0$ non-contractible lines they are

$$
h_{\epsilon_{1} r_{1}+\epsilon_{2} r_{2}-1-2 n, \epsilon_{1} r_{1}+\epsilon_{2} r_{2}-1+2 j} .
$$

The sign $\epsilon_{1}= \pm 1$ indicate whether the leftmost non-contractible line is required to touch the left boundary (for $\epsilon_{1}=+1$, referred to as the "blobbed" sector), or forbidden from doing so (for $\epsilon_{1}=-1$, referred to as the "unblobbed" sector). The sign $\epsilon_{2}$ similarly describe the choice of blobbed/unblobbed sectors at the right boundary, and $n \in \mathbb{N}$. The parameters $r_{1}, r_{2}$ and $r_{12}$ are related to the weight of marked loops through the formulas (59). The case $\beta_{2}=\beta$ and $\beta_{12}=\beta_{1}$ corresponds to the one-boundary loop model (1BLM) [60], where the exponents reduce to [61]

$$
h_{r_{1}, r_{1}+2 j \epsilon_{1}} \text {. }
$$

\subsection{One-boundary case}

We will start by investigating the one-boundary problem. Setting $n=0$, the Hamiltonian we want to study is

$$
H=-u \sum_{i=0}^{m-1} P_{i, i+1}-\sum_{i=m}^{2 L+m-2} E_{i}
$$

When computing the spectrum of this operator, one extra representation theoretical consideration comes into play. Once the number of non-contractible lines (or "strings") at the boundary have been fixed, (63) still acts on them non-trivially by means of permutations. The Hamiltonian can be block-diagonalized with respect to this action by a change of basis that fixes the action of the symmetric group $\mathfrak{S}_{m+j}$ on the $m+j$ strings according to its irreducible representations, the

so-called Specht modules [62]. Since permutations occur between boundary lines and the leftmost bulk line (if any), we will actually deal with $\mathfrak{S}_{m}$ when $j=0$ or $\mathfrak{S}_{m+1}$ when $j>0$.

We can follow the procedure explained in [15] for a related model based on the Brauer algebra. Instead of the usual diagrams on which the Hamiltonian acts, we can consider a tensor product of an 
unlabelled diagram, for which states with exchanged strings are equivalent, and a vector belonging to a basis of a certain Specht module. An operator will act on such a state by the multiplication of diagrams, and by the matrix action in the Specht module of the permutation keeping track of the reordering of strings.

Then the Hamiltonian (63) can be put in a block form, each block indexed by the number of bulk strings and a Young diagram referring to a Specht module. Accordingly the Hilbert space will be decomposed in terms of these subspaces. Numerical diagonalization shows that the lowest eigenvalue of the Hamiltonian always lies in the symmetric representation of the symmetric group, where permutations act as the identity in the Specht module. So for the purpose of computing the leading critical exponents we can restrict to this sector.

\subsubsection{Exponents and relations with the one-boundary loop model}

Diagonalizing numerically the Hamiltonian at different values of $\beta$ and $m$, we have been able to conjecture the analytical form of the $(m+2 j)$-leg critical exponents $h^{m, 0}(j)$. Using the parametrization of the 1BLM, if we define $\beta_{1}$ as

$$
\beta_{1}=\frac{m+\beta}{m+1}
$$

then the exponents are

$$
h^{m, 0}(j)=h_{r_{1}(m), r_{1}(m)+2 j},
$$

where $r_{1}(m)$ is given explicitly by

$$
r_{1}(m)=\frac{1+p}{\pi} \arctan \left(\frac{(1+m) \sin \left(\frac{\pi}{p+1}\right)}{m-(m-1) \cos \left(\frac{\pi}{p+1}\right)}\right) .
$$

Our numerical findings give strong evidence that this result for the exponents does not depend on the coupling $u$ as long as we take it positive (see figure 9). Note also that when $\beta=1$ (whence $\beta_{1}=r_{1}(m)=1$ for all $\left.m\right)$, the exponents are trivial, equal to those of the free boundary case. ${ }^{3}$

In view of this result, which is formally identical to (62) for a particular choice of the parameter $r_{1}(m)$, it is natural to search for a relation between our model and the one-boundary TemperleyLieb algebra. We recall that the latter is defined $[60,61]$ by endowing the Temperley-Lieb algebra with an extra blob operator $b$ acting on the leftmost strand, which we will label $m$ for making easier the connection with our model. By definition, $b$ is idempotent and satisfies the relation

$$
E_{m} b E_{m}=\beta_{1} E_{m},
$$

\footnotetext{
${ }^{3}$ If we think of the case $m=1$, one intuitive argument supporting this triviality goes as follows. Everytime a loop crosses the boundary line, we can think of this configuration as a loop with weight 1 and a straight line times the coupling $-u$ :

$$
\varnothing=-u>0
$$

For general $m$ and $u$ positive we have found the following relation $\lambda_{m}^{0}=-u+\lambda_{m-1}^{0}, \lambda_{m}^{0}$ being the lowest eigenvalues of the Hamiltonian with $m$ boundary lines, which implies the same scaling in $1 / L$ of the two eigenvalues.
} 


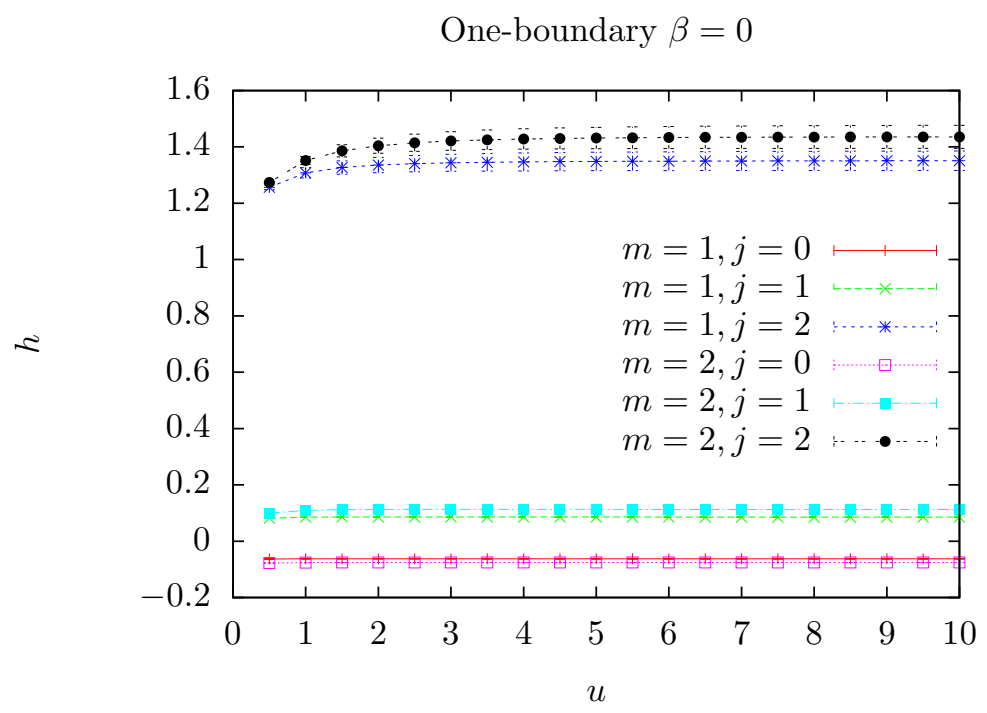

Figure 9: Independence of critical exponents $h$ on the coupling $u$ for $j=0,1,2$ and $m=1,2(n=0)$ in the model with $\beta=0$. The values are computed by finite-size scaling corrections of equation (58) with inclusion of a term of order $1 / L^{2}$, for systems of sizes $L=5$ to $L=9$.

whose geometrical interpretation is that marked loops get a weight $\beta_{1}$.

For the particular case $m=1$ this relationship is manifest. Namely, setting $P_{0}=2 b-1$ and using $b^{2}=b$ we get $\left(P_{0}\right)^{2}=1$ as required. So for a particular choice of the parameter $u$ the boundary interaction is proportional to $b$ indeed. The corresponding weight of boundary loops follows from

$$
E_{1} \frac{1+P_{0}}{2} E_{1}=\frac{1+\beta}{2} E_{1}
$$

where we have used (48). This shows that $\beta_{1}=\frac{1+\beta}{2}$ as in (64). The exponents (65) then follow by invoking the result (62) for the one-boundary loop model [61].

For higher values $m>1$ there is no such mapping to the one-boundary loop model. We can nevertheless derive the results (64)-(66) for an appropriate modification of the Hamiltonian (63). Our argument that (64)-(66) are correct also for the original Hamiltonian (63) is then based on universality: we present numerical evidence that the original and modified Hamiltonians have the same critical exponents.

As a warmup, we first discuss our numerical observation that within the space of Hamiltonians of the form (63), the exponents (65) are independent of the boundary coupling $u$. This should be interpreted in terms of boundary renormalization group flow. The situation is akin to that of the Ising model with a boundary field, where the fixed boundary condition is a stable fixed point, and the free boundary condition an unstable one [63]. At a given non-zero value of the boundary field, 
there is a crossover, and the system flows to the stable fixed point for a sufficiently large system. In our case, the boundary perturbation to the Lagrangian is $u \phi_{r_{1}(m), r_{1}(m)}$, and it drives the system from the $m=0$ to the $m>0$ boundary condition. Since the dimension of the field $\phi_{r_{1}(m), r_{1}(m)}$ is $h_{r_{1}(m), r_{1}(m)}$, by dimensional arguments the relevant length scale in this boundary flow is given by $L^{1-h_{r_{1}(m), r_{1}(m)}}$. We have explicitly checked that curves describing the critical exponents as a function of $u$, collapse after appropriate rescaling in the $\mathfrak{s l}(1 \mid 1)$ case, where we can access large sizes of the system, see figure 10 .

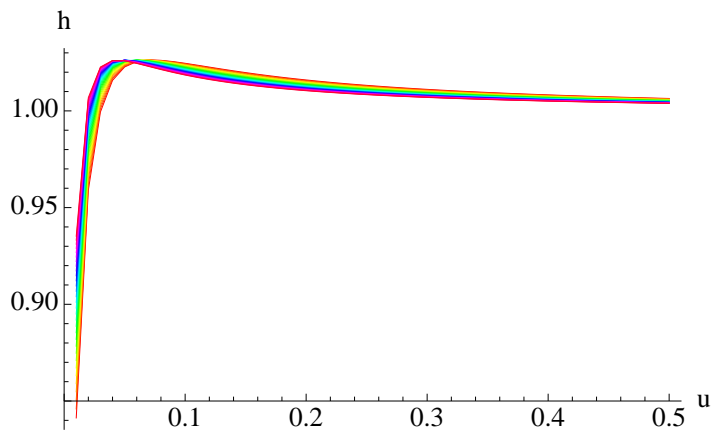

(a)

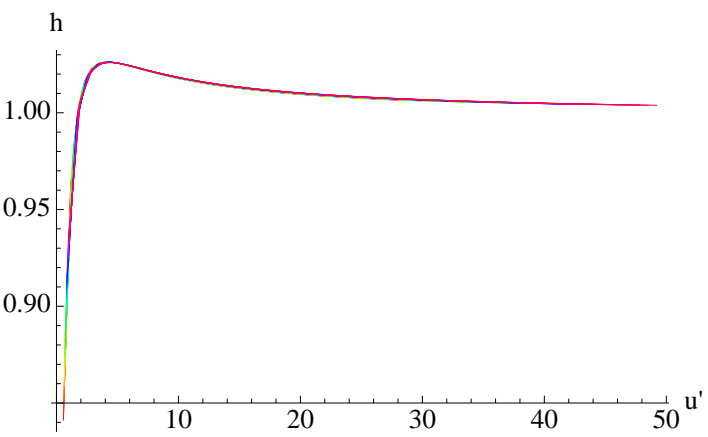

(b)

Figure 10: $h$ vs $u$ in the $\mathfrak{s l}(1 \mid 1)$ chain for sizes of the bulk length $L=45$ to $L=75$ (here in different colors) and $m=1, n=0$. In figure (a) the variable $u$ is not rescaled. In figure (b) we rescale $u$ as $u^{\prime}=L^{1-h_{r_{1}(1), r_{1}(1)}} u$, and we observe a perfect collapse of all curves.

Extending these ideas of universality, we now construct a modified Hamiltonian $\widetilde{H}$ for the lattice model with edge states, which - unlike the original Hamiltonian $H$ of equation (63) - is in precise correspondence with the one-boundary loop model. The key step is to construct an idempotent blob operator $b$ satisfying (67).

In the Hilbert space of our spin chain, the idempotent words of the boundary algebra are given by the Young symmetrizers of the symmetric group $\mathfrak{S}_{m+1}$. In appendix B we show that indeed every symmetrizer satisfies a relation such as (67). The value of $\beta_{1}(64)$ we are interested in is obtained if we take the fully symmetric representation of the symmetric group (see figure 11 for a graphical representation of the relation between the symmetrizer and the blob operator).

Define therefore the standard Young tableau ${ }^{4} t_{1}=$\begin{tabular}{|l|l|l|l|}
\hline & $\cdots$ & $\cdots$ & $m$, and the associated Young
\end{tabular} symmetrizer $b_{t_{1}}=\frac{1}{(m+1) !} \sum_{\sigma \in \mathfrak{S}_{m+1}} \sigma$. It then turns out that $b_{t_{1}}$ satisfies:

$$
E_{m} b_{t_{1}} E_{m}=\frac{m+\beta}{m+1} E_{m} b_{\hat{t}_{1}},
$$

\footnotetext{
${ }^{4}$ In what follows we will adopt the convention of labelling the boxes of a Young diagram of size $m+1$ with the elements of the set $\{0, \ldots, m\}$ when related to the left boundary action of the permutation group
} 


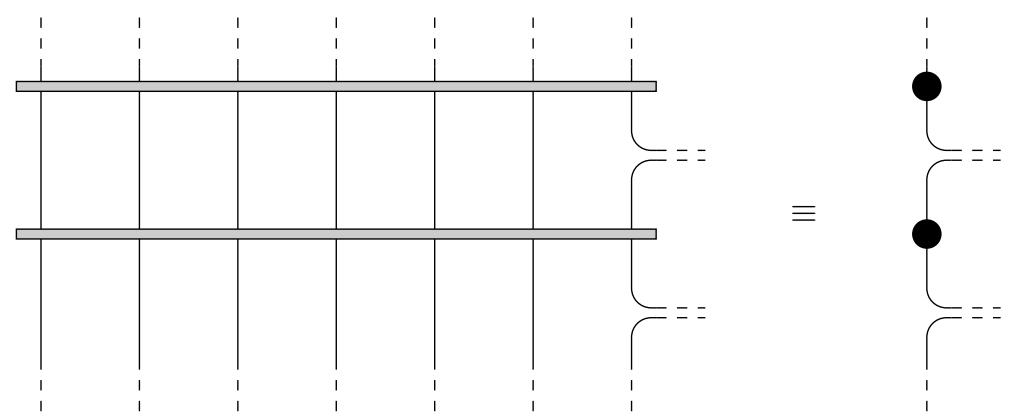

Figure 11: Symmetrization (here represented by horizontal gray bars) over the $m+1$ leftmost strands can be interpreted as blobbing the first bulk strand.

where $\hat{t}_{1}=$\begin{tabular}{|l|l|l|}
\hline 0 & $\cdots$ & $m-1$
\end{tabular}. To prove formula (69) we can use the following simple identity

$$
b_{t_{1}}=\frac{1}{m+1} b_{\hat{t}_{1}}(1+(0, m)+(1, m)+\cdots+(m-1, m)),
$$

$(i, j)$ denoting the transposition of $i$ and $j$, and the relations of the algebra.

Relation (67) now follows with our value of $\beta_{1}(64)$ if we replace $E_{i}$ by $\widetilde{E}_{i}=E_{i} b_{\hat{t}_{1}}$, an operation which does not affect relations of the algebra $\mathcal{A}_{2 L, m, n}$. In other words, the following modified Hamiltonian

$$
\widetilde{H}=-u \frac{1}{(m+1) !} \sum_{\sigma \in \mathfrak{S}_{m+1}} \sigma-\sum_{i=m}^{2 L+m-2} E_{i}
$$

has precisely the form of a one-boundary loop model (the first term being $-u b$ ) and by (69) the weight of a loop marked by $b$ is exactly (64). It follows from (62) that the $2 j$-leg exponents are indeed given by (65).

Obviously the boundary terms in (63) and (71) are different. However, just as the spectrum of (63) was shown numerically to be independent of $u$, it is a natural hypothesis that the critical exponents of $H$ and $\widetilde{H}$ are identical. In table 1 we present strong numerical support for this universality hypothesis.

We remark that for having the universal behaviour described above, it is crucial that the signs in front of the permutation generators are all negative. In this case, adding to the Hamiltonian (63) other terms of the symmetric group than the generators, is a redundant perturbation when they have the same negative coefficients. We expect that taking different couplings $u_{i}$ for each $P_{i, i+1}$ in the boundary Hamiltonian, will in general drive the model to different universality classes according to the relative signs (see also appendix B). In particular, it appears an appealing possibility that each choice of Young symmetrizer in the above construction might lead to a different universality class, but we have not investigated this issue in sufficient detail.

Finally we have numerical evidence that the universality class of $H$ and $\widetilde{H}$ is more general, and 


\begin{tabular}{|c|c|c|c|c|}
\hline$\beta$ & $j$ & $h$ & $h$ & $h_{2,0}(j)$ \\
\hline 0 & 0 & $-0.07606 \pm 0.00010$ & $-0.07615 \pm 0.00015$ & -0.076068 \\
\hline 0 & 1 & $0.1099 \pm 0.0004$ & $0.1090 \pm 0.0006$ & 0.111099 \\
\hline 0 & 2 & $1.339 \pm 0.010$ & $1.321 \pm 0.006$ & 1.298266 \\
\hline 1 & 0 & 0 & 0 & 0 \\
\hline 1 & 1 & $0.33478 \pm 0.00009$ & $0.33478 \pm 0.00009$ & $1 / 3$ \\
\hline 1 & 2 & $2.099 \pm 0.016$ & $2.099 \pm 0.016$ & 2 \\
\hline$\sqrt{2}$ & 0 & $0.01374 \pm 0.00016$ & $0.01302 \pm 0.00021$ & 0.014542 \\
\hline$\sqrt{2}$ & 1 & $0.4384 \pm 0.0011$ & $0.4432 \pm 0.0007$ & 0.438772 \\
\hline$\sqrt{2}$ & 2 & $2.4256 \pm 0.0013$ & $2.4410 \pm 0.0009$ & 2.363001 \\
\hline
\end{tabular}

Table 1: Comparison of critical exponents for the model described by (63), $h$, and for the one described by (71), $\tilde{h}$, listed for different values of $\beta$ and $j$, in a system with $m=2, n=0$. The values are obtained by fitting eigenvalues for bulk sizes $L=6 \rightarrow 10$, using formula (58) with a term of order $1 / L^{2}$, and although these numbers should be equal when $L \rightarrow \infty$, already with these relatively small sizes we find good agreement between the values of the exponents in the model and the exact value predicted in the continuum limit $h_{2,0}(j)$, given in the last column.

that, in particular, the following Hamiltonian also has the same continuum limit:

$$
\widetilde{\widetilde{H}}=b_{\hat{t}_{1}} H b_{\hat{t}_{1}},
$$

corresponding to the case where the $m$ strands on the boundary are fully symmetrized. From an algebraic point of view this version of the model is equivalent to replacing the $m$ copies of the fundamental representation of the superalgebra in the spin chain formulation with a higherdimensional one.

\subsection{Two-boundary case}

We now move to the two-boundary problem, which is obviously much richer.

\subsubsection{New sectors}

The quotient given by the action of the Hamiltonian on the super spin chain discussed previously in section 4.3 allows contraction of boundary lines, introducing a new quantum number in the problem: $k$, the number of couples of left-right boundary lines not contracted. So we will restrict to $(j, k)$ sectors with $j$ and $k$ fixed and the action of TL generators on a pair of strings (non contractible lines) set to zero. See figure 12 for examples of states in different sectors.

A sector of given $k$ appears with multiplicity

$$
\left(\begin{array}{c}
n \\
\mathfrak{m}-k
\end{array}\right)\left(\begin{array}{c}
m \\
\mathfrak{m}-k
\end{array}\right)
$$




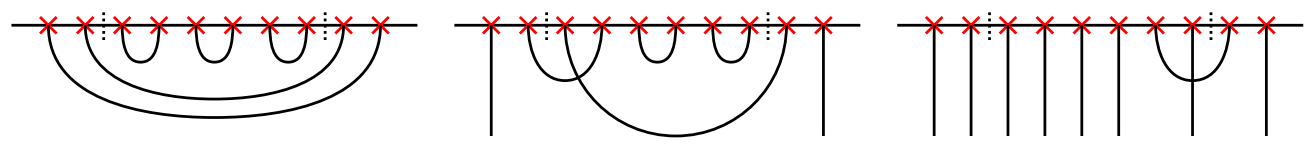

Figure 12: Different sectors in a system with $m=n=2$ and $L=3$. From left to right, $(j=0, k=$ $0),(j=0, k=1),(j=2, k=2)$.

where recall that $\mathfrak{m}=\min (m, n)$. The possible values of $k$ for $j=0,1$ are $k=0,1, \ldots, \mathfrak{m}$. Indeed when $j=0$ or $j=1$ we can always go from a configuration with $k>0$ contracted boundary lines to another with $k-1$ contracted boundary lines by acting with elements of the algebra. In figure 13 , it is shown how we can go from the sector $k=\mathfrak{m}$ to $k=\mathfrak{m}-1$, and it is clear that previous exchange of boundary lines allows contracting up to $\mathfrak{m}$ lines. For $j>1$ meanwhile, there is only the sector $k=\mathfrak{m}$. In this case the boundary lines cannot come on adjacent sites since lines in the bulk cannot cross, as illustrated for $j=2$.

Note that with this definition, the sectors $(j=0, k)$ and $(j=1, k-1)$ are actually the same, and that a state can be regarded as belonging to one or the other sector (see figure 14).

Therefore it remains to study the sectors $(j=0, k=0,1, \ldots, \mathfrak{m})$ and $(j, k=\mathfrak{m})$ when $j>0$. In each such sector the total (bulk plus boundary) number of non-contractible lines is different and correspondingly the value of the exponents will depend on $j+k$. Calling $\ell=|m-n|$, we will refer to the lowest exponent in a sector $(j, k)$ as the $(2 j+2 k+\ell)$-leg exponent, $h^{m, n}(j+k)$. Note anyway that if we would like to look at the lowest exponent when we fix only $j$, this will be present in the sector with the minimum value of $k$ possible.

\subsubsection{Relations with two-boundary loop models and solution}

In the analysis of the one-boundary problem we have found that our model is in the same universality class as a 1BLM with weight of marked loops given by (64). This weight can be obtained by taking as blob operator the Young symmetrizer of the fully symmetric representation of the symmetric group. This identification now turns out to be very useful for solving the two-boundary problem which we recall is described by

$$
H=-u \sum_{i=0}^{m-1} P_{i, i+1}-\sum_{i=m}^{2 L+m-2} E_{i}-v \sum_{i=2 L+m-1}^{2 L+m+n-2} P_{i, i+1} .
$$




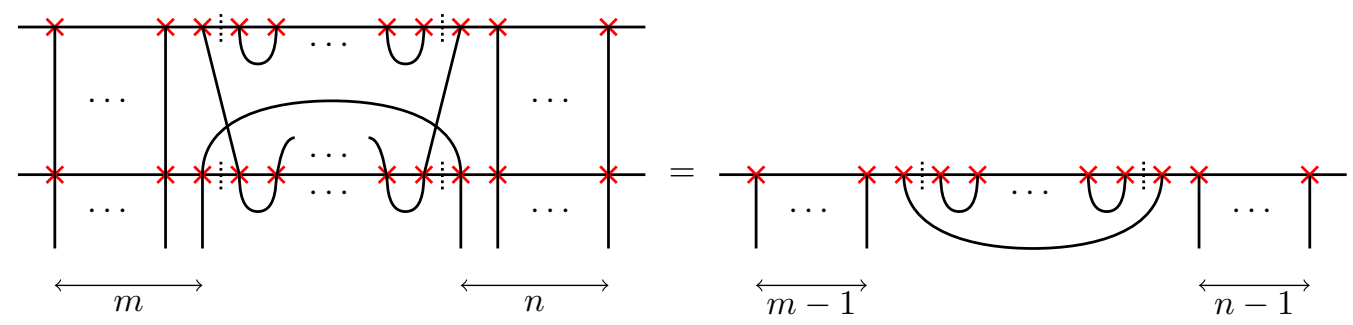

(a)

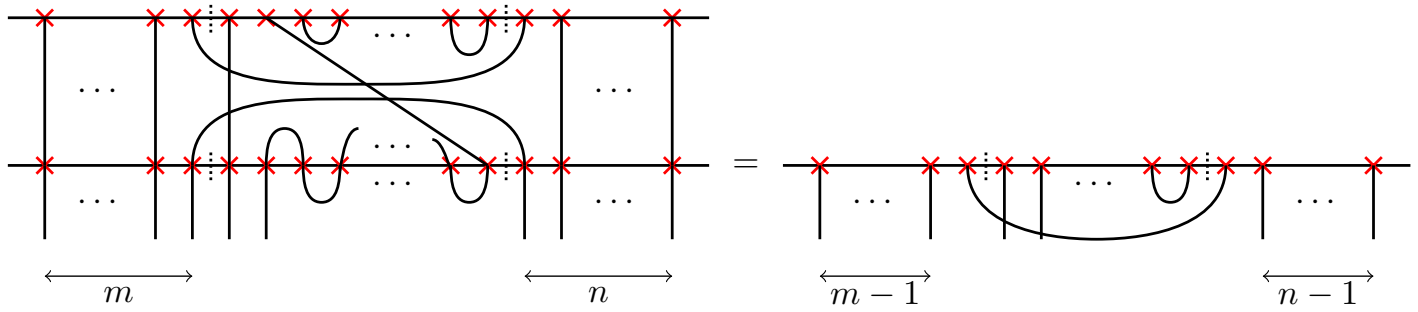

(b)

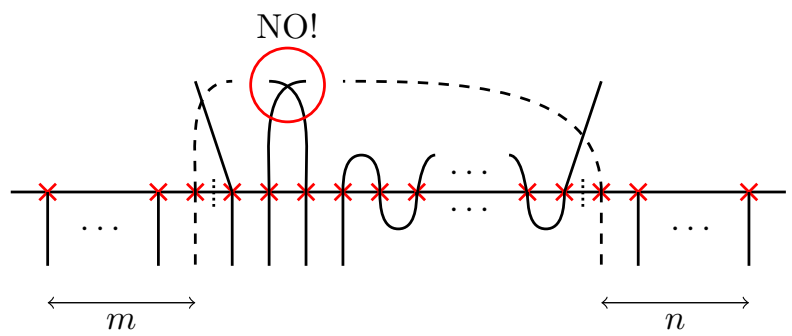

(c)

Figure 13: (a) Starting from the reference (reduced) state on top left at $j=0$ with $k=\mathfrak{m}$, we pass to the state with $k=\mathfrak{m}-1$ on the right upon acting with the word juxtaposed above. (b) Similarly, when $j=1$ multiplication by an element of the algebra allows passing from $k=\mathfrak{m}$ to $k=\mathfrak{m}-1$. (c) When $j=2$, we have to exchange bulk strings in order that the two boundary strings (the dashed lines) can be permuted with the bulk strands, and come on adjacent sites. This is forbidden by the interactions in our model. The situation is similar with higher values of $j$. 


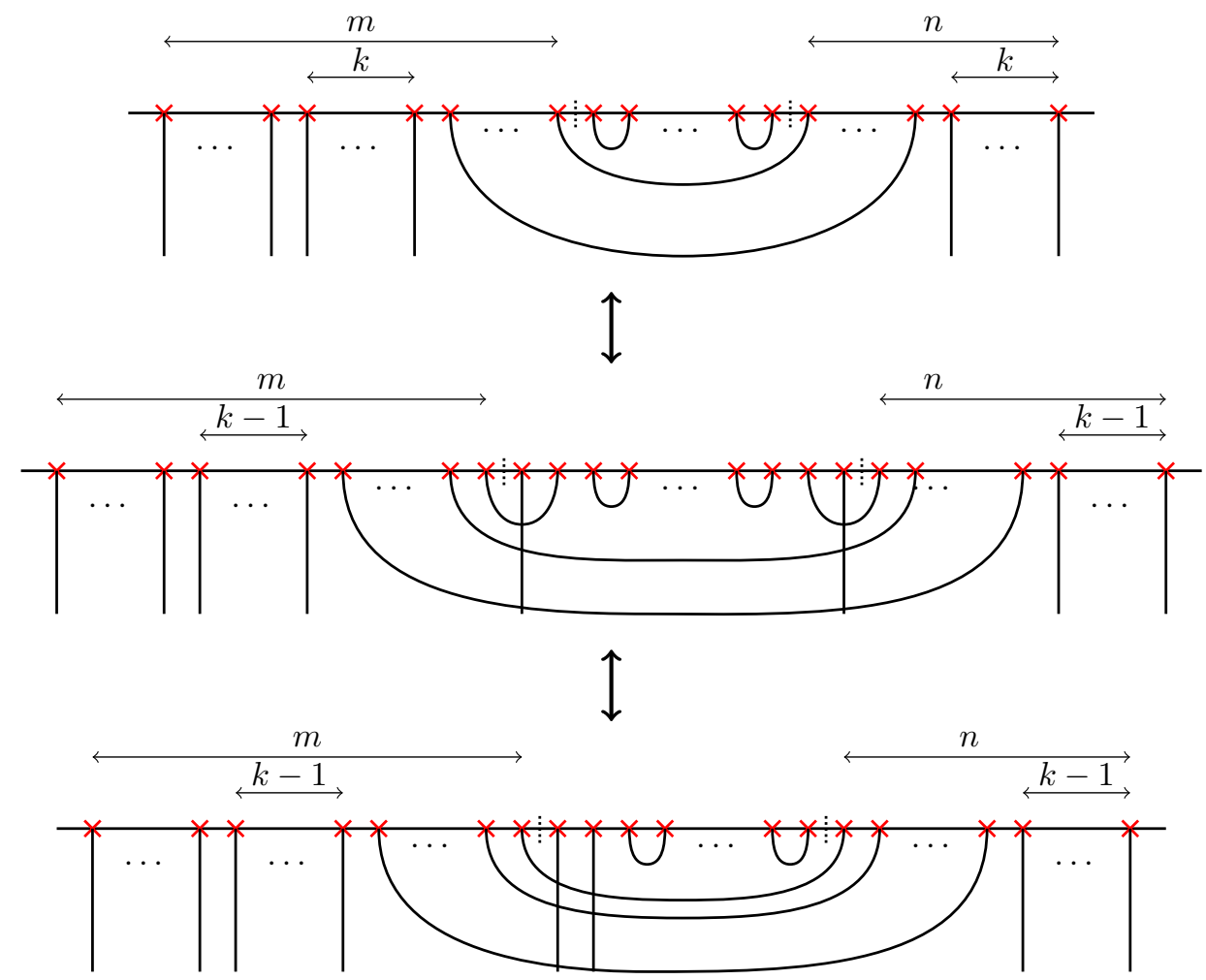

Figure 14: From the reference state of the sector $(j=0, k)$ on top, we can reach the state of the sector $(j=1, k-1)$ on bottom, by the two steps depicted. First, for each boundary we pass one of $k$ lines into the bulk by applying permutations (middle), and then we order the strings using TL generators (we have assumed $m \geq n$ ). Going from bottom to top works in the same manner, and this procedure can be done for every states in the two sectors, which are then equivalent. 
Indeed, by naive analogy, we expect the following Hamiltonian to be in the same universality class as our starting problem (74):

$$
\begin{aligned}
\tilde{H} & =-u \frac{1}{(m+1) !} \sum_{\sigma \in \mathfrak{S}^{\text {left }}} \sigma-\sum_{i=m}^{2 L+m-2} E_{i}-v \frac{1}{(n+1) !} \sum_{\sigma \in \mathfrak{S}^{\mathrm{right}}} \sigma \\
& =-u b_{t_{1}}-\sum_{i=m}^{2 L+m-2} E_{i}-v b_{t_{2}}
\end{aligned}
$$

where we have introduced the symmetric group of the $m+1$ leftmost strands $\mathfrak{S}^{\text {left }}=\mathfrak{S}_{\{0, \ldots, m\}}$, the symmetric group of the $n+1$ rightmost strands $\mathfrak{S}^{\text {right }}=\mathfrak{S}_{\{2 L+m-1, \ldots, 2 L+m+n-1\}}$, as well as the standard Young tableaux $t_{1}=$\begin{tabular}{|l|l|l|l|l|}
\hline 0 & $\cdots$ & $m$
\end{tabular} and $t_{2}=$\begin{tabular}{|l|l|l|}
\hline $2 L+m-1$ & $\cdots$ & $2 L+m+n-1$
\end{tabular} . As before $b_{t}$ stands for the Young symmetrizer related to the tableau $t$.

We have checked numerically the hypothesis that adding these additional permutations to our former Hamiltonian $H$ is a redundant perturbation also in this two-boundary case. See table 2 for a sample of evidence in the case $m=n=2$. As in the one-boundary case, we claim that the results are unchanged as long as $u$ and $v$ are taken positive, as we have verified this numerically (see figure $15)$.

\begin{tabular}{|c|c|c|c|c|c|}
\hline$\beta$ & $j$ & $k$ & $h$ & $\tilde{h}$ & $h_{2,2}(j+k)$ \\
\hline 0 & 0 & 0 & $-0.0026 \pm 0.0010$ & $-0.0041 \pm 0.0013$ & 0 \\
\hline 0 & 0 & 1 & $-0.0026 \pm 0.0010$ & $-0.0041 \pm 0.0013$ & 0 \\
\hline 0 & 0 & 2 & $0.0504 \pm 0.0015$ & $0.0479 \pm 0.0021$ & 0.054734 \\
\hline 0 & 1 & 2 & $0.247 \pm 0.003$ & $0.238 \pm 0.005$ & 0.257230 \\
\hline 0 & 2 & 2 & $1.670 \pm 0.007$ & $1.601 \pm 0.006$ & 1.631564 \\
\hline 1 & 0 & 0 & 0 & 0 & 0 \\
\hline 1 & 0 & 1 & $0.0152 \pm 0.0002$ & $0.0147 \pm 0.0004$ & 0.015906 \\
\hline 1 & 0 & 2 & $0.0700 \pm 0.0012$ & $0.0678 \pm 0.0018$ & 0.073136 \\
\hline 1 & 1 & 2 & $0.3346 \pm 0.0003$ & $0.3346 \pm 0.0003$ & $1 / 3$ \\
\hline 1 & 2 & 2 & $2.118 \pm 0.008$ & $2.118 \pm 0.008$ & 2 \\
\hline
\end{tabular}

Table 2: Comparison of critical exponents for the model described by (74), $h$, and for the one described by (76), $\tilde{h}$, listed for different values of $\beta, j$ and $k$, in a system with $m=2, n=2$. The values are obtained by fitting eigenvalues for bulk sizes $L=4 \rightarrow 8$, and although these numbers should be equal when $L \rightarrow \infty$, already with these relatively small sizes we have good agreement between these two values and with the exact value predicted, given in the last column.

The advantage of studying $\widetilde{H}$ is that it belongs to the two-boundary Temperley-Lieb algebra, the two-boundary analogue of the blob algebra, and for that problem we know how to compute the exponents [59]. In the language of the two-boundary loop model (2BLM), loops marked on the left boundary have weight (64) and those marked on the right boundary

$$
\beta_{2}=\frac{n+\beta}{n+1}
$$




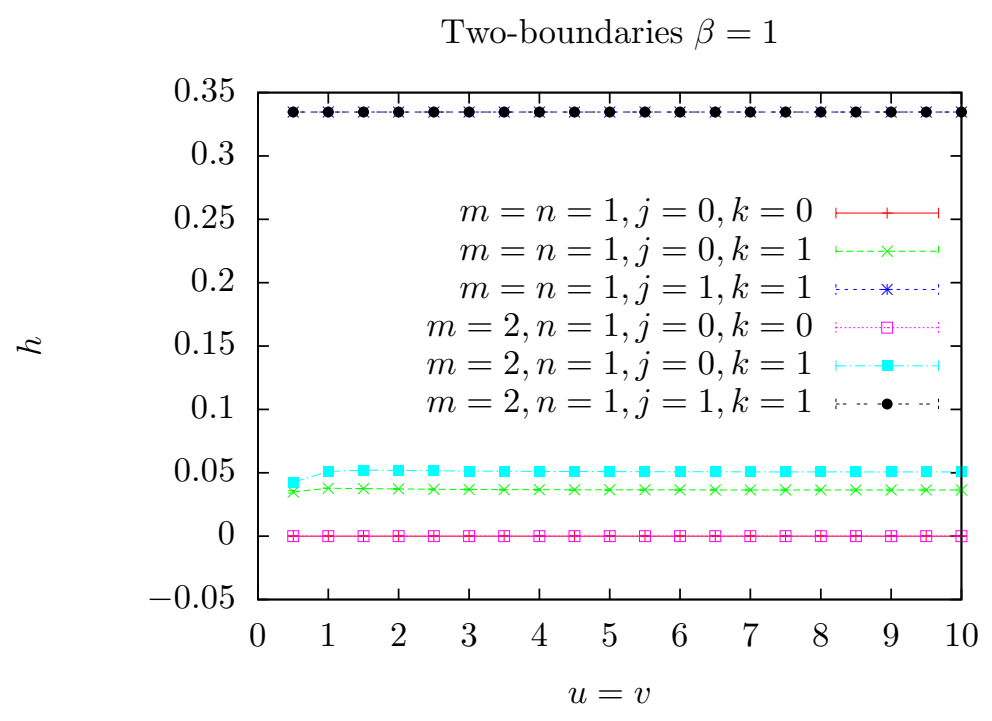

Figure 15: Independence of the first few critical exponents on $u=v$ for $m=n=1$ and $m=2, n=1$ when $\beta=1$. The values are computed by finite size scaling fitting equation (58) with a term of order $1 / L^{2}$, for systems of sizes $L=4$ to $L=8$.

All that remains to be done is to compute $\beta_{12}$, the value of loops marked by both blob operators, as expressed in the following algebraic relation (we can consider for simplicity the situation with $L=1$; in the general case replace $E_{m}$ by $E$ defined in (50)):

$$
E_{m} b_{1} b_{2} E_{m}=\beta_{12} E_{m}
$$

where $b_{1}$ and $b_{2}$ act respectively on the first and second strand labelled $m$ and $m+1$.

So we fix $L=1$, and consider the $j=0$ sector only. Define $\hat{t}_{1}=0|\cdots| m-1, \hat{t}_{2}=$ \begin{tabular}{|l|l|l|l}
$m+2$ & $\cdots$ & $m+n+1$. & If we replace respectively $b_{1}$ and $b_{2}$ in equation (78) with our operators \\
\hline
\end{tabular} $b_{t_{1}}$ and $b_{t_{2}}$, and $E_{0}$ with $\tilde{E}_{m}=E_{m} b_{\hat{t}_{1}} b_{\hat{t}_{2}}$, we would like that the following equation holds for a certain value of $\beta_{12}$ :

$$
\tilde{E}_{m} b_{t_{1}} b_{t_{2}} \tilde{E}_{m}=\beta_{12} \tilde{E}_{m} .
$$

This equation can however only be satisfied and meaningful in our model, if we consider it for a fixed value of $k$, as we will explain below. The sector $(j=0, k)$ of the Hamiltonian (76) will then be described for different $k$ 's by different quotients fixing $\beta_{12}$, which we will now call $\beta_{12}^{k}$ to indicate the $k$-dependence. Taking the quotient as described in section 4.3, we have already implicitly chosen particular values of $\beta_{12}^{k}$, which we are going to compute. We remark that the representation of the two-boundary Temperley-Lieb algebra given by (76) is not faithful in general, and the structure of 
the sectors cannot be directly given from this mapping. However the leading eigenvalues will be present and we explain now how to compute them in the different sectors.

Before showing the general procedure, we will give an example in the case $m=n=2$, where we can understand how the mechanism works. First it is convenient to express $b_{t_{1}}$ and $b_{t_{2}}$ using the following identities:

$$
\begin{aligned}
b_{t_{1}} & =\frac{1}{3} b_{\hat{t}_{1}}(1+(0,2)+(1,2))=\frac{1}{3} b_{\hat{t}_{1}}\left(1+P_{1}\left(1+P_{0}\right)\right) \\
b_{t_{2}}=\frac{1}{3}(1+(3,4)+(3,5)) b_{\hat{t}_{2}} & =\frac{1}{3}\left(1+\left(1+P_{4}\right) P_{3}\right) b_{\hat{t}_{2}} .
\end{aligned}
$$

Then, using the commutation relations of the algebra, we have:

$$
\begin{aligned}
\tilde{E}_{2} b_{t_{1}} b_{t_{2}} \tilde{E}_{2} & =\frac{1}{9} E_{2} b_{\hat{t}_{1}}\left(1+P_{1}\left(1+P_{0}\right)\right)\left(1+\left(1+P_{4}\right) P_{3}\right) b_{\hat{t}_{2}} E_{2}= \\
& =\frac{1}{9}\left((\beta+4) \tilde{E}_{2}+4 b_{\hat{t}_{1}} b_{\hat{t}_{2}} E_{2} P_{1} P_{3} E_{2} b_{\hat{t}_{1}} b_{\hat{t}_{2}}\right) .
\end{aligned}
$$

The first piece of equation (82) is already in the desired form, while the second piece is not proportional to $\tilde{E}_{2}$. As anticipated, we can only achieve this by fixing a sector $k$, and computing the action of the operator $W:=b_{\hat{t}_{1}} b_{\hat{t}_{2}} E_{2} P_{1} P_{3} E_{2} b_{\hat{t}_{1}} b_{\hat{t}_{2}}$ on the states belonging to the sector. The graphical representation of $W$ and $\tilde{E}_{2}$ is depicted in figure 16 .
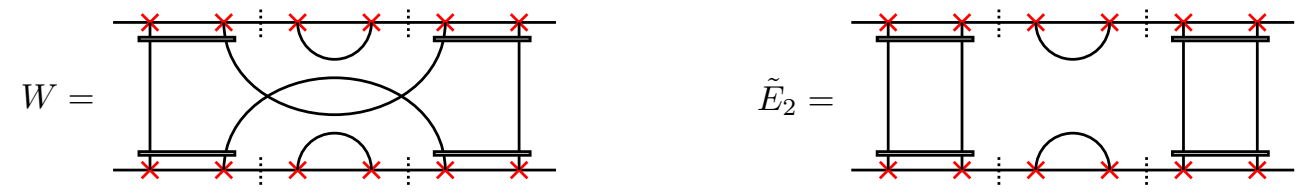

Figure 16: The words $W$ and $\tilde{E}_{2}$ in the diagram representation. As before, the gray bars indicate symmetrization over the lines and $W$ is obtained using the quotient adopted in section 4.4.

Let us first consider the case $k=2$, when no contractions of boundary lines is allowed. It is clear from figure 16 that $W$ acts as zero on every state in this sector, since it diminishes the number of strings from 2 to 1 . So, we have found that every state of the sector $k=2$ is in the kernel of the operator

$$
R:=\left(\tilde{E}_{2} b_{t_{1}} b_{t_{2}}-\beta_{12}^{k}\right) \tilde{E}_{2}
$$

with

$$
\beta_{12}^{2}=\frac{\beta+4}{9} .
$$

We now move to $k=1$. In this sector we have 18 states. They are drawn in figure 17, where we have grouped those related by the action of $b_{\hat{t}_{1}} b_{\hat{t}_{2}}=\left(1+P_{0}+P_{1}+P_{0} P_{1}\right) / 4$. Acting with $\tilde{E}_{2}$ on an arbitrary state will always produce the sum of the four states contained in the box in figure 17 times a constant which can eventually be zero, as when $E_{2}$ acts on two strings. The role of $\tilde{E}_{2}$ is 

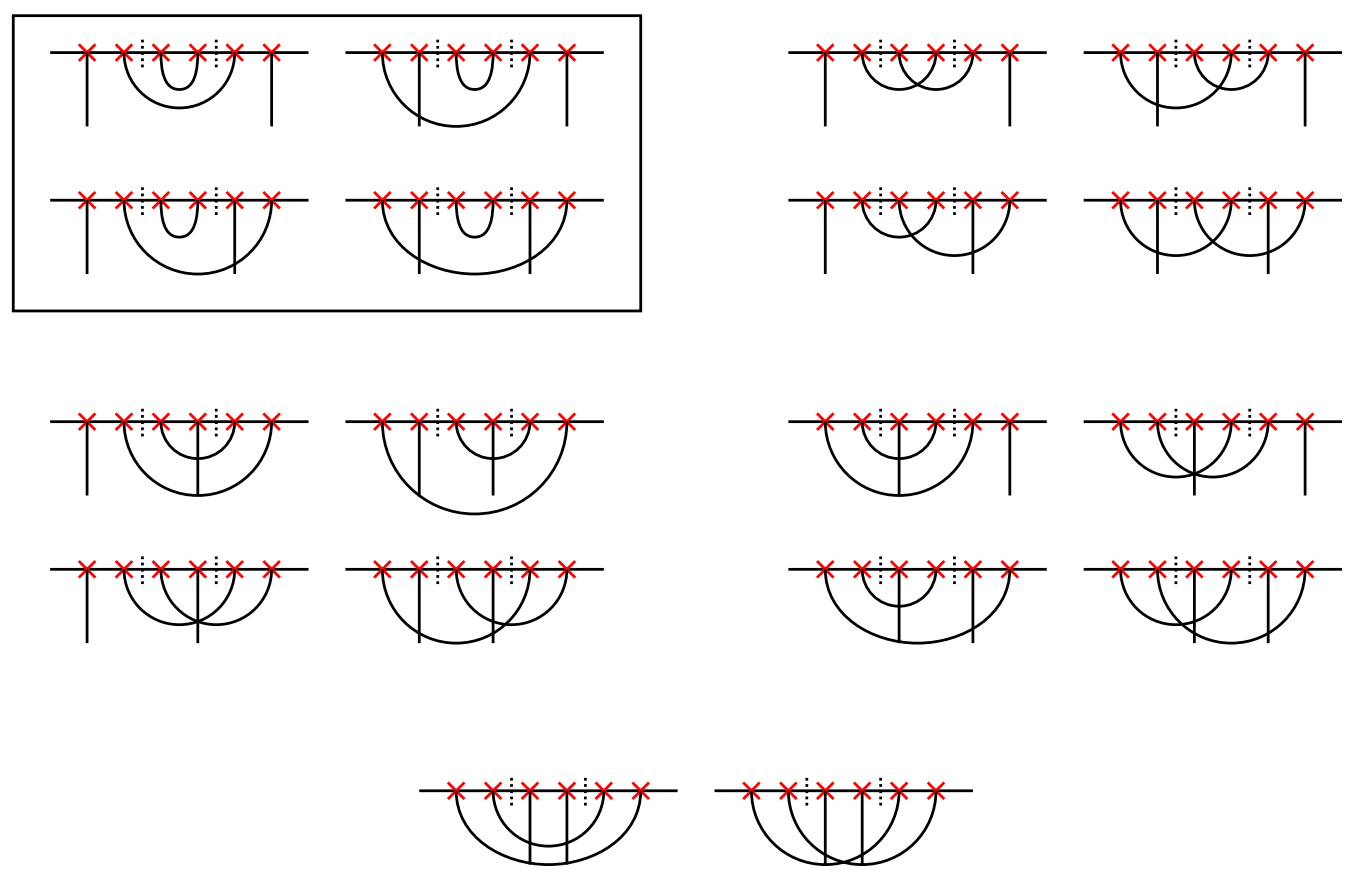

Figure 17: The 18 reduced states of the sector $k=1$, for a system with $m=n=2, L=1$. Those related by the action of $b_{\hat{t}_{1}} b_{\hat{t}_{2}}$ are grouped together. 
indeed to project onto the state in which the boundary strands are fully symmetrized and the bulk ones are contracted. This remark is of crucial importance since it tells us that the action of $R$ is zero on every state, with the same value of $\beta_{12}^{k}$ then characterizing this sector. We can evaluate $R$ on a reference state, say the one on the top left in figure 17 to obtain the value of $\beta_{12}^{k}$. R on this state is zero if:

$$
\beta_{12}^{1}=\frac{2 \beta+6}{9} .
$$

The last case we have to consider is $k=0$, when no strings are left. In this sector we have 6 states depicted in figure 18, where again we have grouped those connected by the action of $b_{\hat{t}_{1}} b_{\hat{t}_{2}}$. As in the previous case, we realize that $\tilde{E}_{2}$ acting on every such states produces the sum of the two
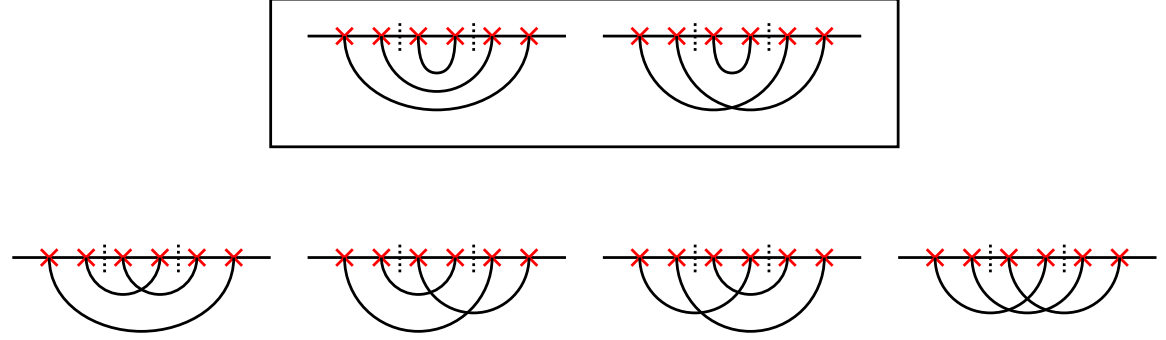

Figure 18: The 6 reduced states of the sector $k=0$, for a system with $m=n=2, L=1$. States on top and on bottom grouped together are related by the action of $b_{\hat{t}_{1}} b_{\hat{t}_{2}}$.

states contained in the box in figure 18 times a constant. Then, evaluating $R$ on a given state gives

$$
\beta_{12}^{0}=\frac{\beta+2}{3} .
$$

Having understood this simple example, we can consider the general case with $m$ and $n$ arbitrary. We first express $b_{t_{1}}$ and $b_{t_{2}}$ in terms of $b_{\hat{t}_{1}}$ and $b_{\hat{t}_{2}}$ :

$$
\begin{aligned}
b_{t_{1}} & =\frac{1}{m+1} b_{\hat{t}_{1}}(1+(0, m)+\cdots+(m-1, m)) \\
& =\frac{1}{m+1} b_{\hat{t}_{1}}\left(1+P_{m-1}\left(\sum_{i=0}^{m-1} \prod_{j=0}^{i-1} P_{m-2-j}\right)\right) \\
b_{t_{2}} & =\frac{1}{n+1}(1+(m+1, m+2)+\cdots+(m+1, m+n+1)) b_{\hat{t}_{2}} \\
& =\frac{1}{n+1}\left(1+\left(\sum_{i=0}^{n-1} \prod_{j=0}^{i-1} P_{m+1+i-j}\right) P_{m+1}\right) b_{\hat{t}_{2}} .
\end{aligned}
$$


Then we arrive at the generalization of formula (82):

$$
\begin{aligned}
\tilde{E}_{m} b_{t_{1}} b_{t_{2}} \tilde{E}_{m}= & \frac{1}{(m+1)(n+1)}\left((\beta+m+n) \tilde{E}_{m}+\right. \\
& \left.+b_{\hat{t}_{1}}\left(\sum_{i=0}^{n-1} \prod_{j=0}^{i-1} P_{m+1+i-j}\right) E_{m} P_{m-1} P_{m+1} E_{m}\left(\sum_{i=0}^{m-1} \prod_{j=0}^{i-1} P_{m-2-j}\right) b_{\hat{t}_{2}}\right)= \\
= & \frac{1}{(m+1)(n+1)}\left((\beta+m+n) \tilde{E}_{m}+m n b_{\hat{t}_{1}} b_{\hat{t}_{2}} E_{m} P_{m-1} P_{m+1} E_{m} b_{\hat{t}_{1}} b_{\hat{t}_{2}}\right),
\end{aligned}
$$

where the last equality holds thanks to the fact that $b_{\hat{t}_{1}}$ can be written as itself times the Young symmetrizer related to the tableau obtained by removing the box numbered $m-1$ in $\hat{t}_{1}$, and the analogous one for $b_{t_{2}}$.

Now, we can generalize our reasoning done for the case $m=2, n=2$. If we redefine the operator

$$
R:=\left(\tilde{E}_{m} b_{t_{1}} b_{t_{2}}-\beta_{12}^{k}\right) \tilde{E}_{m}
$$

then every state of a sector with given $k$ will be in the kernel of $R$ with the same value of $\beta_{12}^{k}$, a consequence of the projection performed by $\tilde{E}_{m}$. The values of $\beta_{12}^{k}$ in the general case $m, n$ are then found to be:

$$
\beta_{12}^{k}= \begin{cases}\frac{(n-k+1)(m+\beta+k)}{(m+1)(n+1)} & \text { if } m \geq n, \\ \frac{(m-k+1)(n+\beta+k)}{(m+1)(n+1)} & \text { if } m<n .\end{cases}
$$

Note that $\beta_{12}^{k}$ is symmetric in exchanging $m, n$, as it should in our problem. The computation of the weights $\beta_{12}^{k}$ in the model described by $\tilde{H}$, equation (76), concludes the analysis of the sector $j=0$.

As explained in section 5.2.1, when we fix $j \geq 1$, we have only to consider $k=\mathfrak{m}$, because if $j=1$, the other values of $k$ are related to corresponding sectors in the $j=0$ case, and when $j>1$, no contraction of strings is allowed. So for $j \geq 1$, the mapping onto the 2BLM can be directly done without any further issue.

The $(2 j+2 k+\ell)$-leg exponents $h_{m, n}(j+k)$ in the model (76) are finally listed in table 3 where we have used the parametrization of $\beta_{1}, \beta_{2}, \beta_{12}^{k}$ in terms of $r_{1}, r_{2}, r_{12}^{k}$ introduced in (59). Recall that $\beta_{1}$ and $\beta_{2}$ are simple functions of $\beta$ and of respectively $m$ and $n$, equations (64) and (77), and that $\mathfrak{m}$ is the minimum between $m$ and $n$. When the number of legs is between $\ell$ and $m+n$, we are in the $j=0$ sector of a $2 \mathrm{BLM}$, and for $k=0, \ldots, \mathfrak{m}$, the exponents $h_{r_{12}^{k}, r_{12}^{k}}$ are computed from our expression of $\beta_{12}^{k}$, equation (93). When we have instead $m+n+2 j$ legs, with $j>0$, we are in the sector $j$ of a $2 \mathrm{BLM}$ which is completely described by the two parameters $\beta_{1}$ and $\beta_{2}$, equations (64) and (77), and the exponents then follow from fusion of the boundary conformal weights of the one-boundary case, the leading exponents in a sector being $h_{r_{1}+r_{2}-1, r_{1}+r_{2}-1+2 j}$. 


\begin{tabular}{|c|c|c|c|}
\hline$j$ & $k$ & $\#$ (legs) & $h^{m, n}(j+k)$ \\
\hline 0 & 0 & $\ell$ & $h_{r_{12}^{0}, r_{12}^{0}}$ \\
0 & 1 & $\ell+2$ & $h_{r_{12}^{1}}, r_{12}^{1}$ \\
0 & 2 & $\ell+4$ & $h_{r_{12}^{2}}, r_{12}^{2}$ \\
$\vdots$ & $\vdots$ & $\vdots$ & $\vdots$ \\
0 & $\mathfrak{m}$ & $m+n$ & $h_{r_{12}^{\mathfrak{m}}, r_{12}^{\mathfrak{m}}}$ \\
1 & $\mathfrak{m}$ & $m+n+2$ & $h_{r_{1}+r_{2}-1, r_{1}+r_{2}-1+2}$ \\
2 & $\mathfrak{m}$ & $m+n+4$ & $h_{r_{1}+r_{2}-1, r_{1}+r_{2}-1+4}$ \\
3 & $\mathfrak{m}$ & $m+n+6$ & $h_{r_{1}+r_{2}-1, r_{1}+r_{2}-1+6}$ \\
$\vdots$ & $\vdots$ & $\vdots$ & $\vdots$ \\
\hline
\end{tabular}

Table 3: Critical exponents for the two-boundary problem.

Invoking the usual universality hypothesis - namely that the critical behaviour is unchanged upon adding permutations other than the generators to the Hamiltonian $H$ - the above expressions completely determine the critical exponents in our original model (74).

Lastly, we have verified that as in the one-boundary case, the version of the model with higher spins on the boundary, $\tilde{\tilde{H}}=b_{\hat{t}_{1}} b_{\hat{t}_{2}} H b_{\hat{t}_{1}} b_{\hat{t}_{2}}$, is in the same universality class as well.

\section{Back to the spin chains spectrum}

In section 4.3 we have suggested that the representation of the quotient algebra $\mathcal{Q}_{2 L, m, n}(M)$ is faithful if the number of states per site in the spin chain is greater or equal to three. Therefore, every irreducible representation of the diagram algebra studied previously should be present in the $\mathfrak{s l}(N+M \mid N)$ spin chains, except when $N=1, M=0$. Whenever the representation is faithful, the eigenvalues of the loop and of the super spin representations are the same, the difference in the Hilbert spaces manifesting itself only in the degeneracies. We can thus use the loop model — which is technically more convenient - to solve the full spin chain problem. This requires understanding all exponents, and calculating their degeneracies. While such a program can easily be carried out for the pure Temperley-Lieb problem [17], in the present case, a lot is still lacking.

One first incomplete aspect of our study is that we have not determined all the geometrical exponents: there are definitely plenty of subleading eigenvalues in a given sector. When a direct mapping onto a Boundary Loop Model is available, we can reinterpret the $(j, k)$ sectors of the edge state model in terms of the blobbed/unblobbed sectors of the BLM and we hence know exactly which conformal weights one finds. Such an exact mapping is possible in the one-boundary system with $m=1$ and $m=0$, for which the conformal weights of primary operators in the sector $j=0$ are $h_{r_{1}, r_{1}}$, and for $j>0$ are $h_{r_{1}, r_{1}+2 j}$ (blobbed sector) and $h_{r_{1}, r_{1}-2 j}$ (unblobbed sector). It is also possible in the two-boundary case with $m=n=1$ if we fix $k=1$, where for $j=0$ we find 
primaries with weight $h_{r_{12}^{1}-2 n, r_{12}^{1}}$ and $n \in \mathbb{Z}$, while for $j>0$ we find $h_{\epsilon_{1} r_{1}+\epsilon_{2} r_{2}-1-2 n, \epsilon_{1} r_{1}+\epsilon_{2} r_{2}-1+2 j}$ with $\epsilon_{1,2}= \pm 1$ and $n \in \mathbb{N}$. But already in the sector $j=k=0$ when $m=n=1$, our algebra gives an unfaithful representation of the two-boundary Temperley-Lieb algebra, since the mapping to a 2BLM involves a quotient version of the latter, and we do not find all the subleading eigenvalues $h_{r_{12}^{0}-2 n, r_{12}^{0}}, n \in \mathbb{Z}$. In general we do not have full control of which subleading eigenvalues appear. However the value of possible eigenvalues we expect should be of the form given by the 2BLM conformal weights with some possible omissions and blobbed/unblobbed sectors mixing, as we have indeed observed in the systems studied numerically. Clearly a deeper algebraic understanding of the problem is needed here to characterize the full spectrum.

The other incomplete aspect is that we lack a clear understanding of the degeneracies. One way to understand these in general is to think of the generating function of eigenvalues (for the super spin chain), which could in principle be computed as the modified partition function of a loop model where non-contractible loops are weighted differently [17]. In algebraic terms, this generating function would be written as a sum over irreducible representations of the algebra to which the Hamiltonian belongs. The multiplicities of summands would then be the dimensions of irreducibles of the commutant algebra in the Hilbert space at hand; the problem is that very little is known in general about this commutant.

Another way to attack the problem of degeneracies is to study the combinatorial aspect of the loop representation of the model, along the lines of [64]. One would then need to develop a theory of combined projectors of the symmetric group (on the boundary) and of the Jones-Wenzl type (in the bulk) in which the degeneracies would be identified with the Markov traces of these projectors. One way or the other, it is clear that further study is needed to finish the program.

For some physical applications [32], only the leading exponents will matter, and the exact knowledge of degeneracies and subleading corrections to scaling does not play a paramount role. However, if these two aspects were completely elucidated, one could construct exact continuum limit partition functions and compute various kinds of exact crossing probabilities, presumably with applications to transport properties in the spin quantum Hall effect. We leave these developments for future work.

Let us finally recall that the $\mathfrak{s l}(1 \mid 1)$ super spin chain-alias symplectic fermions; see appendix A - seems to be the only case where the spin chain representation is not faithful. In particular this manifests itself in the fact that for $m=n$, the spectrum in this chain is equal to that obtained for free $(m=n=0)$ boundary conditions, when the Hamiltonian belongs to the Temperley-Lieb algebra and the critical exponents are those of the conformal field theory of symplectic fermions. Instead, as explicitly showed in table 4 , in the case $m=n \neq 0$, the $\mathfrak{s l}(N \mid N)$ spin chain when $N>1$ possesses an infinity of new exponents which are not contained in symplectic fermion theory. Note that these exponents in general are not rational - a rather unusual feature in conformal field theory. 


\begin{tabular}{|c|c|c|}
\hline Critical Exponents $\beta=0$ & $\mathfrak{s l}(N \mid N)_{N>1}$ & $\mathfrak{s l}(1 \mid 1)$ \\
\hline • $\quad=h_{1,1}=0$ & $\checkmark$ & $\checkmark$ \\
\hline$=h_{1,1}=0$ & $\checkmark$ & $\checkmark$ \\
\hline$\approx h_{0.410,0.410+2} \approx 0.191$ & $\sqrt{ }$ & $x$ \\
\hline$\Lambda \approx h_{0.410,0.410+4} \approx 1.486$ & $\checkmark$ & $x$ \\
\hline 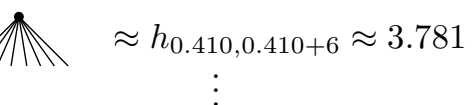 & $\begin{array}{l}\checkmark \\
:\end{array}$ & $\begin{array}{l}x \\
:\end{array}$ \\
\hline
\end{tabular}

Table 4: In the first column, watermelon exponents computed in the geometrical model when $m=n=1$ and $\beta=0$. The number of legs is the coordination of the vertex. In the second and third column we note the presence or not of these exponents in the faithful $(N>1)$ and unfaithful $(N=1)$ representations of the algebra for the $\mathfrak{s l}(N \mid N)$ spin chains.

\section{Conclusion}

In conclusion, we have seen that edge states in super spin chains describe an infinity of conformal invariant boundary conditions for conformal superprojective sigma models. The technology involved to characterize these boundary conditions is definitely involved, and the exponents very far from trivial. How these exponents can be organized in a complete description of the boundary and bulk logarithmic CFT describing the low energy physics of the sigma models remains an open question.

Meanwhile, it is natural to expect that the boundary conditions we have uncovered, together with their edge states description in the quantum spin chains, will play an important role in the description of transitions between plateaus in the spin quantum Hall effect, as well as in the ordinary quantum Hall effect (though in this case a different sigma model would have to be considered) [29]. We hope to get back to this question soon.

Acknowledgments: We thank J. Dubail for very useful comments on the potential relations with boundary Temperley-Lieb algebras. H.S. thanks C. Candu, N. Read, and V. Schomerus for many discussions and earlier collaborations on related matters. This work was supported in part by the Agence Nationale de la Recherche (grants ANR-06-BLAN-0124 and ANR-10-BLAN-0414).

\section{A The $\mathfrak{g l}(1 \mid 1)$ spin chain}

Here we will work out in details the solutions of the model in the simplest case of the $\mathfrak{g l}(1 \mid 1)$ super spin chain, where the Hamiltonian reduces to free fermions and explicit computations can be done. 


\section{A.1 The lie superalgebra $\mathfrak{g l}(1 \mid 1)$}

First we will briefly recall some facts about the Lie superalgebra $\mathfrak{g l}(1 \mid 1)$ and its representation theory [65].

The $\mathfrak{g l}(1 \mid 1)$ Lie superalgebra is defined by two bosonic generators $E, N$ and two fermionic ones $F^{-}, F^{+}$, and its superdimension is zero. $E$ is central, and the other defining relations are

$$
\left[N, F^{ \pm}\right]= \pm F^{ \pm}, \quad\left\{F^{-}, F^{+}\right\}=E .
$$

In particular $N$ counts the number of fermions.

When dealing with the irreducible representations of superalgebras, we have to distinguish between typical or atypical representations. For $\mathfrak{g l}(1 \mid 1)$ typical representations are the two-dimensional representations $\langle e, n\rangle, e \neq 0, n \in \mathbb{R}$, which read in matrix notation:

$$
N=\left(\begin{array}{cc}
n-1 & 0 \\
0 & n
\end{array}\right) \quad F^{-}=\left(\begin{array}{ll}
0 & 1 \\
0 & 0
\end{array}\right) \quad F^{+}=\left(\begin{array}{cc}
0 & 0 \\
e & 0
\end{array}\right) \quad E=e \mathbf{1}_{2} .
$$

The state annihilated by $F^{-}$is a boson. Atypical representations are the one-dimensional irreducible representations $\langle n\rangle$, which have $F^{+}=F^{-}=E=0, N=n$.

Other finite-dimensional representations that occur are indecomposable, not fully reducible. They are composites of atypical constituents linked by the action of $F^{ \pm}$. We denote by $\mathcal{P}_{n}$ the indecomposable four-dimensional representation playing the role of the projective cover of $\langle n\rangle$, that is it can never appear as a subrepresentation of a larger indecomposable and contains $\langle n\rangle$.

The spin chain (37) is built with the fundamental $V=\langle 1,1 / 2\rangle$ and the dual $V^{\star}=\langle-1,1 / 2\rangle$. The (graded) tensor product of representations is given by the following formulas:

$$
\begin{aligned}
\left\langle e_{1}, n_{1}\right\rangle \otimes\left\langle e_{2}, n_{2}\right\rangle & = \begin{cases}\mathcal{P}_{n_{1}+n_{2}-1} & \text { if } e_{1}+e_{2}=0, \\
\left\langle e_{1}+e_{2}, n_{1}+n_{2}\right\rangle \oplus\left\langle e_{1}+e_{2}, n_{1}+n_{2}-1\right\rangle & \text { otherwise. }\end{cases} \\
\langle e, n\rangle \otimes \mathcal{P}_{m} & =\langle e, n+m+1\rangle \oplus 2 \cdot\langle e, n+m\rangle \oplus\langle e, n+m-1\rangle .
\end{aligned}
$$

Then we have for $l_{1} \neq l_{2}$ :

$$
V^{\otimes l_{1}} \otimes\left(V^{\star}\right)^{\otimes l_{2}} \simeq \bigoplus_{p=0}^{1} \bigoplus_{i=0}^{l_{1}-1} \bigoplus_{j=0}^{l_{2}-1}\left(\begin{array}{c}
l_{1}-1 \\
i
\end{array}\right)\left(\begin{array}{c}
l_{2}-1 \\
j
\end{array}\right)\left\langle l_{1}+l_{2}, i+j+2-\frac{l_{1}+l_{2}}{2}+p\right\rangle .
$$

This is the decomposition of the Hilbert space (37) when the number of extra representations on the two boundaries of the spin chain is different. It involves $\mathfrak{g l}(1 \mid 1)$ 2-dimensional representations, since changing the order of representations in the spin chain leads to isomorphic spaces. When instead $l_{1}=l_{2}=l$

$$
\left(V \otimes V^{\star}\right)^{\otimes l} \simeq \bigoplus_{i=1-l}^{l-1}\left(\begin{array}{c}
2 l-2 \\
l-1+i
\end{array}\right) \mathcal{P}_{i}
$$

this corresponding to the decomposition of the Hilbert space in terms of 4-dimensional representations when we have the same number of representations at the two boundaries of the chain. 


\section{A.2 Free fermions formulation}

Denote by $f_{i}, f_{i}^{\dagger}$ the fermionic operators of $\mathfrak{g l}(1 \mid 1)$ acting on $V$ at site $i$, and by $\bar{f}_{i}, \bar{f}_{i}^{\dagger}$ those acting on $V^{\star}$. They satisfy the anti-commutation relations $\left\{f_{i}, f_{j}^{\dagger}\right\}=\delta_{i j},\left\{\bar{f}_{i}, \bar{f}_{j}^{\dagger}\right\}=-\delta_{i j}$, where the presence of the additional minus sign implies that the Hilbert space has indefinite inner product. To deal with ordinary fermions $c_{i}, c_{i}^{\dagger}$ that satisfy $\left\{c_{i}, c_{j}^{\dagger}\right\}=\delta_{i j}$, we define $c_{i}=f_{i}$ and $c_{i}^{\dagger}=f_{i}^{\dagger}$ if we have $V$ on site $i$; if we have $V^{\star}$ on site $i$ define instead $c_{i}=\bar{f}_{i}$ and $c_{i}^{\dagger}=-\bar{f}_{i}^{\dagger}$.

$P_{i}$ and $E_{i}$ defined in section 4.3 can be written in terms of these fermions as:

$$
\begin{aligned}
P_{j, j+1} & =\left(1-\left(c_{j}^{\dagger} c_{j}-c_{j+1}^{\dagger} c_{j}-c_{j}^{\dagger} c_{j+1}+c_{j+1}^{\dagger} c_{j+1}\right)\right), \quad j=0, \ldots, m-1 \\
E_{j} & =(-1)^{j+m+1}\left(c_{j}^{\dagger} c_{j}-c_{j+1}^{\dagger} c_{j}+c_{j}^{\dagger} c_{j+1}-c_{j+1}^{\dagger} c_{j+1}\right), \quad j=m, \ldots, 2 L+m-2 \\
P_{j, j+1} & =-\left(1-\left(c_{j}^{\dagger} c_{j}-c_{j+1}^{\dagger} c_{j}-c_{j}^{\dagger} c_{j+1}+c_{j+1}^{\dagger} c_{j+1}\right)\right), \quad j=2 L+m-1, \ldots, 2 L+m+n-2 .
\end{aligned}
$$

One can check by hand that the relations (46)-(49) are satisfied. The Hamiltonian (40) in this representation becomes quadratic in the fermionic operators:

$$
\begin{aligned}
H= & u \sum_{j=0}^{m-1}\left(c_{j}^{\dagger} c_{j}-c_{j+1}^{\dagger} c_{j}-c_{j}^{\dagger} c_{j+1}+c_{j+1}^{\dagger} c_{j+1}\right)+ \\
& +\sum_{j=m}^{2 L+m-2}(-1)^{j+m}\left(c_{j}^{\dagger} c_{j}-c_{j+1}^{\dagger} c_{j}+c_{j}^{\dagger} c_{j+1}-c_{j+1}^{\dagger} c_{j+1}\right)+ \\
& -v \sum_{j=2 L+m-1}^{2 L+m+n-2}\left(c_{j}^{\dagger} c_{j}-c_{j+1}^{\dagger} c_{j}-c_{j}^{\dagger} c_{j+1}+c_{j+1}^{\dagger} c_{j+1}\right)-m u+n v .
\end{aligned}
$$

\section{A.2.1 Diagonalization procedure}

Rewrite (102) as

$$
H=\sum_{i, j} c_{i}^{\dagger} A_{i j} c_{j}-m u+n v,
$$

where $A$ is an appropriate $(2 L+m+n) \times(2 L+m+n)$ real non-symmetric tridiagonal matrix.

The problem of diagonalizing a Hamiltonian of the form (103) is a well-known problem, solved by Lieb, Mattis and Schultz in [66]. We introduce by a canonical transformation the fermions $\eta_{k}, \eta_{k}^{\dagger}$, in terms of which $H$ is diagonal:

$$
H=\sum_{k} \Lambda_{k}\left(\eta_{k}^{\dagger} \eta_{k}-\frac{1}{2}\right) .
$$

However the equations involved in the diagonalization procedure even in the simplest case $m=1, n=0$ are rather complicated, and instead we will proceed diagonalizing the matrix numerically. This task we can accomplish more efficiently by using a closed formula for the characteristic polynomial of $A$ for generic values of $m$ and $n$. Indeed we have found that $P_{L}^{m, n}(u, v, x)=$ 
$\operatorname{Det}\left(A-x \mathbf{1}_{2 L+m+n}\right)$ has the following expression:

$$
\begin{aligned}
& P_{L}^{m, n}(u, v, x)=x(-1)^{L+n} u^{m-1} v^{n-1}\left\{U_{L+1}\left(1-\frac{x^{2}}{2}\right) \cdot\right. \\
& \quad \cdot\left(U_{m+1}\left(1-\frac{x}{2 u}\right)\left(p_{1}(u, v, x) U_{n}\left(1+\frac{x}{2 v}\right)+p_{2}(u, v, x) U_{n+1}\left(1+\frac{x}{2 v}\right)\right)+\right. \\
& \left.\quad+U_{m}\left(1-\frac{x}{2 u}\right)\left(p_{3}(u, v, x) U_{n}\left(1+\frac{x}{2 v}\right)+p_{1}(-v, u,-x) U_{n+1}\left(1+\frac{x}{2 v}\right)\right)\right)+ \\
& +U_{L}\left(1-\frac{x^{2}}{2}\right) . \\
& \quad \cdot\left(U_{m+1}\left(1-\frac{x}{2 u}\right)\left(q_{1}(u, v, x) U_{n}\left(1+\frac{x}{2 v}\right)+q_{2}(u, v, x) U_{n+1}\left(1+\frac{x}{2 v}\right)\right)+\right. \\
& \left.\left.\quad+U_{m}\left(1-\frac{x}{2 u}\right)\left(q_{3}(u, v, x) U_{n}\left(1+\frac{x}{2 v}\right)+q_{1}(-v, u,-x) U_{n+1}\left(1+\frac{x}{2 v}\right)\right)\right)\right\}
\end{aligned}
$$

where $U_{i}(z)$ are the Chebyshev polynomials of the second kind,

$$
U_{i}(z)=\frac{\sin \left((i+1) \cos ^{-1}(z)\right)}{\sqrt{1-z^{2}}}
$$

and the coefficients $p$ 's and $q$ 's are simple polynomials depending only on $u, v$ and $x$ :

$$
\begin{aligned}
& p_{1}(u, v, x)=u\left(x^{4}+2 x^{3} v-x^{2}(v+2)-4 x v+v\right) \\
& p_{2}(u, v, x)=-x\left(x^{2}-2\right) u v \\
& p_{3}(u, v, x)=x\left(x^{4}+2 x^{3}(v-u)-x^{2}(4 u v+u+v+2)+4 x(u-v)+9 u v+u+v\right) \\
& q_{1}(u, v, x)=-u\left(v-x\left(x^{2}-3\right)\left(x^{3}+2 x^{2} v-x(v+1)-2 v\right)\right) \\
& q_{2}(u, v, x)=-x\left(x^{4}-4 x^{2}+3\right) u v \\
& q_{3}(u, v, x)=-x\left(v\left(x\left(x\left(4 u x-2 x^{2}+x+8\right)-17 u-3\right)-6\right)+14 u\right) \\
& \left.\quad+x\left(x^{2}-3\right)\left(x\left(-2 u x+u+x^{2}-1\right)+2 u\right)+u+v\right)
\end{aligned}
$$

Formula (105) seems to not help in the analytic determination of the eigenvalues apart from the trivial case $m=0, n=0$, but it is remarkable that the dependence on $L, m, n$ is organized in terms of Chebyshev polynomials, and further it allows efficient numerical diagonalization of the Hamiltonian. The presence of Chebyshev polynomials does not come as a surprise since it is an old result that these polynomials are related to the discrete Laplacian [67], and our formula is in this sense a generalization of that result.

We remark that the matrix $A$ introduced above is actually not diagonalizable when $m=n$, and it has Jordan cells of rank 2. This is linked to the indecomposable nature of the conformal field theory describing the $\mathfrak{g l}(1 \mid 1)$ chain $[19,25]$. 


\section{A.3 Spectrum of the Hamiltonian and continuum limit}

We would like now to determine the spectrum of the Hamiltonian (104). We define the vacuum $|0\rangle$ as the state with no particles: $\eta_{k}|0\rangle=0$ for every $k$, whose energy is $-\frac{1}{2} \sum \Lambda_{k}$. Define $I^{-}$and $I^{+}$ as the set of values of $k$ 's such that the real part of the corresponding eigenvalues are respectively negative and positive. The ground state $|\Omega\rangle$ is obtained by filling the single particle states with negative energy up to the Fermi surface, $|\Omega\rangle=\prod_{k \in I^{-}} \eta_{k}^{\dagger}|0\rangle$, and its energy is

$$
E_{0}=\frac{1}{2}\left(\sum_{k \in I^{-}} \Lambda_{k}-\sum_{k \in I^{+}} \Lambda_{k}\right) .
$$

We can calculate the conformal weights $h_{i}$ from finite-size effects using formula (58). Ultimately with the information gained from finite-size scaling, we can compute for given boundary conditions $m, n$ :

$$
Z_{m, n}=\lim _{L \rightarrow \infty} \operatorname{Tr} e^{-\beta\left(H-E_{0}(L) L\right)}=\operatorname{Tr} q^{L_{0}-c / 24}=\sum_{i} q^{h_{i}-c / 24},
$$

where Tr stands for an ordinary trace on the vector space, not a supertrace, and $q$ is as usual the modular parameter. This corresponds to a modified partition function, with antiperiodic boundary conditions in the time direction [17].

\section{A.3.1 Free boundary conditions}

We start by analyzing the simplest and already known case of the Temperley-Lieb chain with open boundary conditions $(m=n=0)$. In this case indeed formula (105) reduces to

$$
P_{L}^{0,0}(x)=(-1)^{L+1} x^{2} U_{L-1}\left(1-\frac{x^{2}}{2}\right)
$$

and its roots are given by $x=0$ with multiplicity two, and the roots of $U_{L-1}\left(1-\frac{x^{2}}{2}\right)$ :

$$
x=2 \sin \left(\frac{\pi k}{2 L}\right), \quad k= \pm 1, \ldots, \pm(L-1) .
$$

The eigenvectors corresponding to these eigenvalues can also be easily computed.

With these values of $\Lambda_{k}$ determined, we can use the Euler-Maclaurin formula and equation (58) to verify that the central charge of the model is $c=-2$ (using that $v_{s}=2$ for $\left.p=1\right)$ :

$$
\begin{aligned}
\frac{E_{0}}{2 L} & =-\frac{1}{2 L} \sum_{k=1}^{L-1} 2 \sin \left(\pi \frac{k}{2 L}\right)=\frac{1-\cot \left(\frac{\pi}{4 L}\right)}{2} \\
& \simeq-\frac{2}{\pi}-\frac{1}{2 L}+\frac{\pi}{6(2 L)^{2}} .
\end{aligned}
$$


To compute the partition function in the free boundary case, we note first that $h_{0}=0$. Then since $L / \pi \sum_{i=1}^{p} \Lambda_{k_{i}} \sim \sum_{i=1}^{p} k_{i}$ for large $L, p \ll L$, the exponents are all integers and their computation is rather simple. Indeed it boils down to compute all the ways we can build the integer $\sum_{i=1}^{p} k_{i}$ using at most two equal $k_{i}$ 's (one associated to the action of $c_{k_{i}}^{\dagger}$, the other of $c_{-k_{i}}$ ). If we write

$$
Z_{0,0}=q^{-c / 24} \sum_{i=0}^{\infty} a_{i} q^{i}
$$

and use the notation $\lambda=\left(\lambda_{1}^{n_{1}}, \ldots, \lambda_{k}^{n_{k}}\right) \vdash i$ for the partition of size $i, a_{i}$ is given by

$$
a_{i}=4 \sum_{\substack{\lambda \vdash i \\ n_{j}=1,2}} \prod_{j=1}^{k} 2^{\delta_{n_{j}, 1}} .
$$

The factor 4 comes from having two zero $\Lambda_{k}$ 's and parts occurring only once have a weight two because they can come from the action of $c_{k_{i}}^{\dagger}$ or $c_{-k_{i}}$. As customary when dealing with the generating function of partitions, we are not able to give an expression in closed form for $a_{i}$ but we can do this for the generating function:

$$
Z_{0,0}=\prod_{h=0}^{\infty}\left(1+2 q^{h}+q^{2 h}\right)=2 \frac{\theta_{2}(\tau)}{\eta(\tau)}=\operatorname{det}\left(-\mathcal{D}_{A, N}\right)
$$

where $\mathcal{D}_{A, N}$ is the Laplacian on the strip with Neumann boundary conditions in the space direction and antiperiodic boundary conditions in the time direction.

Finally we note that this result is in agreement with the expression of the partition function as sums of Virasoro characters (recall however that the modules involved are not simple but indecomposable for this theory [19]):

$$
\begin{aligned}
Z_{0,0} & =\sum_{j=0}^{\infty}(2 j+1) \frac{q^{h_{1,1+2 j}}-q^{h_{1,-1-2 j}}}{\eta(\tau)} \\
& =2 \frac{q^{h_{1,1}}}{\eta(\tau)}+2 \sum_{j=1}^{\infty} \frac{q^{h_{1,1+2 j}}+q^{h_{1,1-2 j}}}{\eta(\tau)} .
\end{aligned}
$$

\section{A.3.2 Generic boundary conditions}

We now turn to the discussion of generic values of $m, n$. In this case we do not have an analytic expression for the eigenvalues of the Hamiltonian and so we proceed numerically using the results presented in A.2.1, and we extract the finite-size scaling by fitting energies for different sizes of the system. We have studied chains up to size $2 L=24$.

Define:

$$
r=1-\frac{2}{\pi} \arctan \left(\frac{|m-n|}{1+m+n+2 m n}\right),
$$


where the absolute value comes from the symmetry upon exchanging $m$ and $n$. We find that the partition function of the $\mathfrak{g l}(1 \mid 1)$ chain is given by replacing the exponents $h_{1,1+k}$ by $h_{r, r+k}$ in (122):

$$
Z_{m, n}=2 \frac{q^{h_{r, r}}}{\eta(\tau)}+2 \sum_{j=1}^{\infty} \frac{q^{h_{r, r+2 j}}+q^{h_{r, r-2 j}}}{\eta(\tau)} .
$$

Now we will discuss the relation between this spin chain and the geometrical model at $\beta=0$. We have pointed out in section 4.4 and 6 that the $\mathfrak{g l}(1 \mid 1)$ representation is not faithful, so not all the eigenvalues of the Hamiltonian in the geometrical formulation are present here. We now wish to make this observation more quantitative.

In the $\mathfrak{g l}(1 \mid 1)$ spin chain the conformal weights appearing in (124) are $h_{r, r \pm 2 j}, j=0,1, \ldots$ If we compute explicitly $\beta_{12}^{k}$ from our expression of exponents in the geometrical model (table 3 ) when $\beta=0$, we find that the function $r$ defined in (123) coincides with $r_{12}^{0}$, so we interpret $h_{r, r}$ as the $\ell$-leg exponent. Further $r_{12}^{1}=2-r$ and then $h_{r, r+2}$ is the $(\ell+2)$-leg exponent. When we look at other exponents the situation is different for the one-boundary and the two-boundary cases.

For the one-boundary case $n=0$, in the spin chain we always find the eigenvalues of the Hamiltonian corresponding to the fully symmetric (and also to the alternating) representation of the symmetric group acting onto the strings, where the lowest eigenvalues lie. So, $h_{r, r+2 j}$ are the $(m+2 j)$-legs critical exponents we found in the loop representation.

When we look at the two-boundary problem, the lowest eigenvalues in the geometrical model for $(j=0, k=0)$ and $(j=0, k=1)$ are present in the spin chain, as already noted. However, for the other sectors this is no more the case, and the conformal weights $h_{r, r \pm 2 j}, j>1$, appear as subleading eigenvalues in the sectors of the geometrical model.

We remark that for $m=n$ the spectrum in the scaling limit of the $\mathfrak{g l}(1 \mid 1)$ spin chain is the same as that in the free boundary case, and the multiplicity of the exponents $h_{r, r \pm 2 j}$ is 4 while for $m \neq n$ is 2. This can be interpreted from the analysis of the tensor product of representations of $\mathfrak{g l}(1 \mid 1)$ given in (A.1). These numbers correspond to the multiplicities of modules of the commutant of $\mathfrak{g l}(1 \mid 1)$, the Walled Brauer algebra, and are present also in our case.

As a last remark, we interpret this result in terms of the continuum calculation presented in section 2.3 for the $\mathbb{C P}^{0 \mid 1}$ sigma model, which the spin chain introduced in this section should discretize. Indeed we note that when $\mu=0$ formula (19) can be rewritten as equation (124), where $r=1-2 \lambda$.

\section{B Young symmetrizers as blob operators}

Irreducible representations of the symmetric group $\mathfrak{S}_{m+1}$, the Specht modules, are obtained by a corresponding Young symmetrizer, indexed by a standard Young tableau $t$ of size $m+1$, where in each box of the diagram there are numbers from the set $\{0, \ldots, m\}$. Young symmetrizers $b_{t}$ have 
the property of being idempotent if properly normalized, $b_{t}^{2}=b_{t}$. More than that, they satisfy also the other relation of the blob algebra:

$$
E_{m} b_{t} E_{m}=\beta_{1}^{t} E_{m} b_{\hat{t}},
$$

if we replace $E_{m}$ with $\tilde{E}_{m}=E_{m} b_{\hat{t}}$. $\hat{t}$ is a standard Young tableau of size $m$, obtained from $t$ by removing the box labelled $m$. So, one way to realize the blob algebra is taking a Young symmetrizer acting on the $m$ boundary lines and the leftmost bulk strand. The weight of blobbed loops in this construction will be indexed by the Young tableau and is given by:

$$
\beta_{1}^{t}=\frac{\prod_{x \in Y(\hat{t})} \operatorname{hook}(x)}{\prod_{x \in Y(t)} \operatorname{hook}(x)}\left(\beta+L_{t}(m)-A_{t}(m)\right)
$$

$Y(t)$ is the Young diagrams to which the tableau $t$ is associated, hook $(x)$ is the hook length of the box $x, L_{t}(m)$ is the number of boxes to the left of the box numbered $m$, and $A_{t}(m)$ is the number of boxes above the box numbered $m$.

One can verify formulas (125)-(126) by computing the first cases, doable by hand. For example, for the case of the Young symmetrizer of the symmetric representation, our formula is consistent with the result of equation (64). If we take the alternating representation instead, we have $\beta_{1}=$ $(\beta-m) /(m+1)$. When one looks at 1BLM realizations of this algebra, however the interpretation of the critical behaviour of such a model can be difficult for negative $\beta_{1}$, since phenomena like level crossings appear, as has been discussed in the context of the boundary chromatic polynomial [68].

We defer to future work further comments about the generalizations of this construction to the case of two-boundary Temperley-Lieb, which is definitely a more involved task.

\section{References}

[1] K. B. Efetov, "Supersymmetry and theory of disordered metals," Adv. Phys. 32 (1983) $53-127$.

[2] N. Read Unpublished, 1987.

[3] H. Weidenmüller, "Single electron in a random potential and a strong magnetic field," Nucl. Phys. B 290 (1987) 87-110.

[4] M. R. Zirnbauer, "Conformal field theory of the integer quantum Hall plateau transition," arXiv:hep-th/9905054.

[5] M. Bocquet, D. Serban, and M. R. Zirnbauer, "Disordered 2d quasiparticles in class D: Dirac fermions with random mass, and dirty superconductors," Nucl. Phys. B 578 no. 3, (2000) $628-680$. 
[6] E. Witten, "Perturbative Gauge Theory as a String Theory in Twistor Space," Commun. Math. Phys. 252 no. 1-3, (2004) 189-258, arXiv:hep-th/0312171.

[7] N. Mann and J. Polchinski, "Finite Density States in Integrable Conformal Field Theories," arXiv:hep-th/0408162.

[8] N. Mann and J. Polchinski, "Bethe ansatz for a quantum supercoset sigma model," Phys. Rev. D 72 no. 8, (2005) 30, arXiv:hep-th/0508232.

[9] O. Aharony, O. Bergman, D. L. Jafferis, and J. Maldacena, " $\mathrm{N}=6$ superconformal Chern-Simons-matter theories, M2-branes and their gravity duals," J. High Energy Phys. 2008 no. 10, (2008) 091, arXiv:0806.1218 [hep-th].

[10] V. Schomerus and H. Saleur, "The $G L(1 \mid 1)$ WZW-model: From supergeometry to logarithmic CFT," Nucl. Phys. B $\mathbf{7 3 4}$ no. 3, (2006) 221-245, arXiv:hep-th/0510032.

[11] T. Quella, V. Schomerus, and T. Creutzig, "Boundary spectra in superspace $\sigma$-models," $J$. High Energy Phys. 2008 no. 10, (2008) 024-024, arXiv:0712.3549 [hep-th].

[12] G. Gotz, T. Quella, and V. Schomerus, "Tensor products of psl(2|2) representations," arXiv:hep-th/0506072.

[13] V. Mitev, T. Quella, and V. Schomerus, "Principal chiral model on superspheres," J. High Energy Phys. 2008 no. 11, (2008) 086, arXiv:0809.1046 [hep-th].

[14] C. Candu, T. Creutzig, V. Mitev, and V. Schomerus, "Cohomological Reduction of Sigma Models," J. High Energy Phys. 2010 (2010) 1-39, arXiv:1001.1344 [hep-th].

[15] C. Candu and H. Saleur, "A lattice approach to the conformal $O S p(2 S+2 \mid 2 S)$ supercoset sigma model. Part I: Algebraic structures in the spin chain. The Brauer algebra," Nucl. Phys. B 808 no. 3, (2009) 441-486, arXiv:0801.0430 [hep-th].

[16] C. Candu, V. Mitev, T. Quella, H. Saleur, and V. Schomerus, "The sigma model on complex projective superspaces," J. High Energy Phys. 2010 no. 2, (2010) 59, arXiv:0908.0878 [hep-th].

[17] N. Read and H. Saleur, "Exact spectra of conformal supersymmetric nonlinear sigma models in two dimensions," Nucl. Phys. B 613 no. 3, (2001) 409-444, arXiv:hep-th/0106124.

[18] N. Read and H. Saleur, "Enlarged symmetry algebras of spin chains, loop models, and S-matrices," Nucl. Phys. B 777 no. 3, (2007) 263-315, arXiv:cond-mat/0701259.

[19] N. Read and H. Saleur, "Associative-algebraic approach to logarithmic conformal field theories," Nucl. Phys. B 777 no. 3, (2007) 316-351, arXiv:hep-th/0701117. 
[20] P. A. Pearce, J. Rasmussen, and J.-B. Zuber, "Logarithmic minimal models," J. Stat. Mech.:Theory E 2006 no. 11, (2006) P11017, arXiv:hep-th/0607232v3.

[21] P. A. Pearce, J. Rasmussen, and S. P. Villani, "Solvable critical dense polymers on the cylinder," J. Stat. Mech.:Theory E 2010 no. 02, (2010) P02010, arXiv:0910.4444v3 [hep-th].

[22] N. Read private communication, 1993.

[23] M. R. Zirnbauer, "Toward a theory of the integer quantum Hall transition: Continuum limit of the Chalker-Coddington model," J. Math. Phys. 38 no. 4, (1997) 2007-2036.

[24] J. Kondev and J. B. Marston, "Supersymmetry and localization in the quantum Hall effect," Nucl. Phys. B 497 no. 3, (1997) 639-657, arXiv:cond-mat/9612223.

[25] T. Creutzig, T. Quella, and V. Schomerus, "Branes in the GL(1|1) WZNW model," Nuclear Physics B 792 no. 3, (2008) 257 - 283, arXiv:0708.0583v1 [hep-th].

[26] T. Creutzig, "Geometry of branes on supergroups," Nucl. Phys. B 812 no. 3, (2009) 301-321, arXiv:0809.0468 [hep-th].

[27] D. Khmel'nitskii and M. Yosefin, "Conductance fluctuations of 2D electrons in a strong magnetic field," Surf. Sci. 305 no. 1-3, (1994) 507-510.

[28] D. Maslov and D. Loss, "Edge-state transport and conductance fluctuations in the metallic phase of the quantum Hall regime," Phys. Rev. Lett. 71 no. 25, (1993) 4222-4225.

[29] S. Xiong, N. Read, and A. Stone, "Mesoscopic conductance and its fluctuations at a nonzero Hall angle," Phys. Rev. B 56 no. 7, (1997) 3982-4012, arXiv: cond-mat/9701077.

[30] A. Pruisken, "Super universality of the quantum Hall effect and the "large $N$ picture" of the $\vartheta$ angle," Int. J. Theor. Phys. 48 (2009) 1736-1765, arXiv:0811. 3299 [cond-mat.mes-hall].

[31] I. Gruzberg, A. Ludwig, and N. Read, "Exact Exponents for the Spin Quantum Hall Transition," Phys. Rev. Lett. 82 no. 22, (1999) 4524-4527, arXiv: cond-mat/9902063.

[32] R. Bondesan, I. Gruzberg, J. L. Jacobsen, H. Obuse, and H. Saleur In preparation, 2011.

[33] H. G. Kausch, "Curiosities at $c=-2$," arXiv:hep-th/9510149.

[34] H. Kausch, "Symplectic fermions," Nucl. Phys. B 583 no. 3, (2000) 513-541, arXiv:hep-th/0003029. 
[35] F. Wegner, "Four-loop-order $\beta$-function of nonlinear $\sigma$-models in symmetric spaces," Nucl. Phys. B 316 no. 3, (1989) $663-678$.

[36] C. Candu, J. L. Jacobsen, N. Read, and H. Saleur, "Universality classes of polymer melts and conformal sigma models," J. Phys. A: Math. Gen. 43 (2010) 142001, arXiv:0908.1081 [cond-mat.stat-mech].

[37] M. Bershadsky, "PSL $(n \mid n)$ sigma model as a conformal field theory," Nucl. Phys. B 559 no. 1-2, (1999) 205-234, arXiv:hep-th/9902180.

[38] F. D. M. Haldane, "Nonlinear Field Theory of Large-Spin Heisenberg Antiferromagnets: Semiclassically Quantized Solitons of the One-Dimensional Easy-Axis Néel State," Phys. Rev. Lett. 50 no. 15, (1983) 1153-1156.

[39] I. Affleck, "The quantum Hall effects, $\sigma$-models at $\Theta=\pi$ and quantum spin chains," Nucl. Phys. B 257 (1985) 397-406.

[40] N. Read and S. Sachdev, "Some features of the phase diagram of the square lattice SU(N) antiferromagnet," Nucl. Phys. B 316 no. 3, (1989) 609 - 640.

[41] M. Zirnbauer, "Towards a theory of the integer quantum Hall transition: From the nonlinear sigma model to superspin chains," Ann. Phys. (Berlin) 506 no. 7-8, (1994) 513-577, arXiv: cond-mat/9410040.

[42] H. N. V. Temperley and E. H. Lieb, "Relations between the percolation and colouring problem and other graph-theoretical problems associated with regular planar lattices: some exact results for the percolation problem," P. Roy. Soc. Lond. A Mat. 322 (1971) 251-280.

[43] T.-K. Ng, "Edge states in antiferromagnetic quantum spin chains," Phys. Rev. B 50 no. 1, (1994) 555-558.

[44] S. Qin, T.-K. Ng, and Z.-B. Su, "Edge states in open antiferromagnetic Heisenberg chains," Phys. Rev. B 52 no. 17, (1995) 12844-12848, arXiv: cond-mat/9502047.

[45] S. Coleman, "More about the massive Schwinger model," Ann. Phys. (New York) 101 no. 1, (1976) $239-267$.

[46] A. Ram, "Characters of Brauer's centralizer algebras," Pac. J. Math. 169 no. 1, (1995) $173-200$.

[47] W.F. Doran, D.B. Wales, P.J. Hanlon, "On the semisimplicity of the Brauer centralizer algebras," J. Algebra 211 no. 2, (1999) 647-685. 
[48] P. Martin, Potts models and related problems in statistical mechanics. Series on advances in statistical mechanics. World Scientific, 1991.

[49] J. De Gier and A. Nichols, "The two-boundary Temperley-Lieb algebra," J. Algebra 321 no. 4, (2009) 1132-1167, arXiv:math/0703338.

[50] V. Jones, "A quotient of the affine Hecke algebra in the Brauer algebra," L'Enseignement Mathématique 40 no. 2, (1994) 313-344.

[51] F. H. L. Essler, H. Frahm, and H. Saleur, "Continuum limit of the integrable superspin chain," Nucl. Phys. B $\mathbf{7 1 2}$ no. 3, (2005) 513-572, arXiv: cond-mat/0501197.

[52] M. T. Batchelor and C. M. Yung, "Integrable vertex and loop models on the square lattice with open boundaries via reflection matrices," Nucl. Phys. B 435 (1995) 430.

[53] S. Chen, Y. Wang, and F.-C. Pu, "Integrability of spin chain with boundary impurities," Phys. Lett. A 247 no. 1-2, (1998) 176-182.

[54] B. Nienhuis, "Exact Critical Point and Critical Exponents of O(n) Models in Two Dimensions," Phys. Rev. Lett. 49 no. 15, (1982) 1062-1065.

[55] M. P. M. den Nijs, "A relation between the temperature exponents of the eight-vertex and q-state Potts model," J. Phys. A: Math. Gen. 12 no. 10, (1979) 1857.

[56] H. W. J. Blöte and M. P. Nightingale, "Conformal invariance, the central charge, and universal finite-size amplitudes at criticality," Phys. Rev. Lett. 56 no. 7, (1986) 742-745.

[57] I. Affleck, "Universal term in the free energy at a critical point and the conformal anomaly," Phys. Rev. Lett. 56 no. 7, (1986) 746-748.

[58] J.-F. Richard and J. L. Jacobsen, "Character decomposition of Potts model partition functions, I: Cyclic geometry," Nucl. Phys. B 750 (2006) 250-264.

[59] J. Dubail, J. L. Jacobsen, and H. Saleur, "Conformal two-boundary loop model on the annulus," Nucl. Phys. B 813 no. 3, (2009) 430-459, arXiv:0812.2746 [math-ph].

[60] P. Martin and H. Saleur, "On an algebraic approach to higher dimensional statistical mechanics," Commun. Math. Phys. 158 no. 1, (1993) 155-190, arXiv:hep-th/9208061.

[61] J. L. Jacobsen and H. Saleur, "Conformal boundary loop models," Nucl. Phys. B 788 no. 3, (2008) 137-166, arXiv:math-ph/0611078.

[62] B. E. Sagan, The Symmetric Group: Representations, Combinatorial Algorithms, and Symmetric Functions, Second Edition. Springer, 2001. 
[63] I. Affleck and A. Ludwig, "Universal noninteger ground-state degeneracy in critical quantum systems," Phys. Rev. Lett. 67 no. 2, (1991) 161-164.

[64] J. L. Jacobsen and H. Saleur, "Combinatorial aspects of boundary loop models," J. Stat. Mech.:Theory E 2008 no. 01, (2008) P01021, arXiv:0709.0812 [math-ph].

[65] G. Gotz, T. Quella, and V. Schomerus, "Representation theory of $\mathrm{sl}(2 \mid 1)$," J. Algebra 312 no. 2, (2007) 829-848, arXiv:hep-th/0504234.

[66] E. Lieb, T. Schultz, and D. Mattis, "Two soluble models of an antiferromagnetic chain," Ann. Phys. (New York) 16 no. 3, (1961) 407-466.

[67] P. L. Nash, "Chebyshev Polynomials and Quadratic Path Integrals.," J. Math. Phys. no. 27, (1986) 2963.

[68] J. L. Jacobsen and H. Saleur, "Boundary chromatic polynomial," J. Stat. Phys. 132 (2008) 707-719, arXiv:0803.2665 [math-ph]. 